\title{
Magnetoencephalography Coherence Source Imaging in Dyslexia: Activation of Working Memory Pathways
}

\author{
Alfred Mansour ${ }^{1 *}$, Susan M. Bowyer ${ }^{2,3,4}$, Annette E. Richard1, John E. Moran'2, \\ Laszlo A. Erdodi ${ }^{5}$, Amy Olszewski ${ }^{1,6}$, Lesley Pawluk ${ }^{1,7}$, Daniel Jacobson ${ }^{\mathbf{8}}$, Kelly Vogt ${ }^{1}$, \\ Aimee M. Moore', Renée Lajiness-0'Neill1,7 \\ ${ }^{1}$ Department of Psychology, Eastern Michigan University, Ypsilanti, USA \\ ${ }^{2}$ Neuromagnetism Lab, Department of Neurology, Henry Ford Hospital, Detroit, USA \\ ${ }^{3}$ Wayne State University, Detroit, USA \\ ${ }^{4}$ Oakland University, Rochester, USA \\ ${ }^{5}$ Department of Psychiatry, Geisel School of Medicine at Dartmouth, Hanover, USA \\ ${ }^{6}$ Department of Psychiatry, SUNY Upstate Medical University, Syracuse, USA \\ ${ }^{7}$ Department of Psychiatry, Division of Neuropsychology, Henry Ford Hospital, Detroit, USA \\ ${ }^{8}$ United States Air Force, USMS, Biloxi, USA \\ Email: *amansour@emich.edu, rlajines@gmail.com
}

Received 13 August 2014; revised 5 September 2014; accepted 29 September 2014

Copyright (C) 2014 by authors and Scientific Research Publishing Inc.

This work is licensed under the Creative Commons Attribution International License (CC BY).

http://creativecommons.org/licenses/by/4.0/

cc) (i)

Open Access

\section{Abstract}

This study investigates the functional connectivity of neuronal networks critical for working memory in individuals with dyslexia by means of magnetoenchephalographic (MEG) coherence imaging. Individuals with dyslexia showed an early onset of activation in anterior cortical regions (precentral gyrus and the superior frontal gyrus), which differed from controls where activation initiated in posterior cortical regions (supramarginal gyrus and superior temporal gyrus). Further, individuals with dyslexia showed lower brain activity in the right superior temporal gyrus and right middle temporal gyrus than controls during a spatial working memory (SWM) task. In contrast, during a verbal working memory (VWM) task, individuals with dyslexia showed lower activity in the right insular cortex and right superior temporal gyrus and higher, likely compensatory, activity in the right fusiform gyrus, left parahippocampal gyrus, and left precentral gyrus. When performing a SWM task, individuals with dyslexia showed significantly lower coherent activity and synchronization in 1) right frontal connectivity, 2) right fronto-temporal connectivity, 3) left and right frontal connectivity, 4) left temporal and right frontal connectivity, and 5) left occipital and right frontal connectivity. MEG coherence source imaging (CSI) by frequency bands showed lower

${ }^{*}$ Corresponding author.

How to cite this paper: Mansour, A., Bowyer, S. M., Richard, A. E., Moran, J. E., Erdodi, L. A., Olszewski, A., Pawluk, L., Jacobson, D., Vogt, K., Moore, A. M., \& Lajiness-O’Neill, R. (2014). Magnetoencephalography Coherence Source Imaging in Dyslexia: Activation of Working Memory Pathways. Psychology, 5, 1879-1910. http://dx.doi.org/10.4236/psych.2014.516193 
mean coherence values in individuals with dyslexia compared to controls for each frequency range during the SWM task. In contrast, during the VWM task, individuals with dyslexia showed higher coherent low frequency $(3-15 \mathrm{~Hz})$ and lower coherent high frequency $(30-45 \mathrm{~Hz})$ synchronization than control subjects. Logistic regression of coherent activity by group membership was significant, with an overall predictive success of $84.4 \%$ (88.9\% for controls and $\mathbf{7 7 . 8 \%}$ for dyslexia). Coherence between the right lateral orbitofrontal and middle orbitofrontal gyri pair substantially contributed to group membership. The results suggest a pattern of aberrant connectivity as evidenced by the early onset and reliance on prefrontal cortical areas, the differential activation of fronto-temporal brain systems, and an altered pattern of functional connectivity in the frontotemporal pathways mediating these behaviors.

\title{
Keywords
}

\author{
Dyslexia, Reading Disorder, Learning Disorder, Magnetoencephalography, Functional Brain \\ Imaging
}

\section{Introduction}

An estimated 5\% to $17 \%$ of school age children have significant difficulties learning to read despite average or above average intelligence, adequate educational opportunities, and environmental support (Shaywitz et al., 1998; Vellutino, Fletcher, Snowling, \& Scanlon, 2004). Thus defined, developmental dyslexia has far-reaching societal and economic consequences and often results in life-long emotional and psychological distress for the individuals suffering from this disorder and their families. While there is general agreement as to the neuropsychological contributions to the development of dyslexia, there is little consensus as to the precise neurobiological mechanisms and brain circuits that may be involved.

Indefrey \& Levelt (2004) integrated the results of several functional brain mapping studies and modalities and suggested that there are specific time intervals and subprocesses that take place in reading. Visual object recognition and conceptualization occur at 0 - 175 milliseconds post stimulus presentation and involve occipital and ventrotemporal regions. First, there is a visual feature analysis around the occipital midline at approximately 100 milliseconds. In non-dyslexia about 50 milliseconds later, the activation becomes lateralized to the left occipital-temporal cortex if letters or words are involved (word recognition). The selection of the semantic-syntactic representation occurs at 175 - 250 milliseconds and is associated with activation of the left middle and superior temporal gyri. Phonological processing (word decoding) occurs at 250 - 330 milliseconds and involves the activation of left middle and superior temporal gyri and the parietal cortical regions (supramarginal gyrus, angular gyrus). Oral output or the articulation of a word or speech sound occurs after 330 milliseconds and results in activation of Broca's area in the left inferior frontal gyrus and bilateral sensorimotor areas. These MEG localizations and latencies have been corroborated using several different MEG analysis techniques (Bowyer et al., 2004; Simos et al., 2002).

Functional deficits in semantic fluency and written language are associated with the left occipital-temporal activation, which is selectively diminished in individuals with dyslexia for words but not for faces (Tarkiainen, Helenius, \& Salmelin, 2003). Similar differences have been observed in word reading versus picture-naming tasks between individuals with dyslexia and neurotypical readers, suggesting the deficits may be more specific for reading (Trauzettel-Klosinski, Dürrwächter, Klosinski, \& Braun, 2006). During a word recognition task using MEG imaging, children with dyslexia showed an initial activation in the left basal temporal regions (fusiform gyrus) followed by activation of the right temporal-parietal regions (including the angular, superior temporal and supramarginal gyri). Neurotypical controls, in contrast, show an initial activation in the left basal temporal regions followed by activation of the left temporal-parietal regions (Simos et al., 2000). These results suggest an anomalous functional connectivity between the left basal temporal and the left temporal-parietal regions in individuals with dyslexia during word recognition. Instead of relying on specialized left hemispheric brain systems for reading as did controls, individual with dyslexia activate homotopic, less efficient regions in the right hemisphere for word recognition. 
MEG spatiotemporal brain activation patterns or signatures associated with a psuedoword rhyme-matching task similarly show a reduced or slowed left temporal-parietal (posterior part of superior temporal, angular and supramarginal gyri) activation and increased activity in the homotopic region on the right hemisphere of dyslexic individuals (Papanicolaou et al., 2003). To better assess the phonological basis of this difference in brain activation in individuals with dyslexia, investigators used an auditory discrimination task (Wehner, Ahlfors, \& Mody, 2007) and demonstrated that the reduced MEG left hemispheric activation in individuals with dyslexia correlated with the phonological difficulty of the task. Further, the individuals with dyslexia did not benefit from the degree of phonological contrast compared to the control group. Taken together, these results suggest that the phonological and orthographic deficits of dyslexia are due to the aberrant functional connections of the brain areas that mediate reading, as opposed to the dysfunction of a specific brain region.

Reading remediation studies further demonstrate anomalous neuronal connections in dyslexia, and there is a functional re-organization of neuronal pathways with intervention. Eighty hours of intensive remedial reading instruction over a two-month period improved reading skills and resulted in increased left superior temporal gyrus activation in dyslexics performing a psuedoword reading task (Simos et al., 2002; Simos et al., 2006). Similar benefits of reading remediation have been demonstrated with MEG on a word-reading task, in which they observed a reduced aberrant right temporal-parietal activation and lateralized activation to the left hemisphere following treatment (Sarkari et al., 2002). Treatment effects of Fast ForWord, hypothesized to ameliorate temporal processing deficits, were demonstrated by magnetoencephalography in a child with dyslexia using four paradigms (Lajiness-O'Neill, Akamine, \& Bowyer, 2007): Word/Non-word Reading (NW), Grapheme-to-Phoneme Matching (GP), Verbal Working Memory (VWM), and Spatial Working Memory (SWM). Shifts in brain activation from right inferior frontal and temporal to left frontal, bilateral supramarginal, and transverse temporal regions occurred during GP. During NW, shifts progressed from 1) right or bilateral anterior and superior to 2) left, inferior frontal, to 3) left, superior posterior temporoparietal, to 4) left, inferior, posterior temporooccipital regions. Reading and written language improvements were noted in passage comprehension and spelling.

Coherence analysis of MEG data provides a functional map of the brain areas activated in any millisecond time segment and the correlational probability (certainty) of forming such functional connections. As with EEG, MEG coherence analysis has routinely been applied to the sensor space, analyzing which channels having similar frequency content. With advances in the technology, MEG coherence imaging has been extended to source space and applied to the study of motor control (Belardinelli et al., 2007) and the lateralization of temporal lobe epilepsy (Elisevich et al., 2011). However, thus far, MEG coherence imaging in source space has not been widely applied to other clinical population such as dyslexia, where a better understanding of the functional neuronal networks and the neuroplastic changes that underlie this disorder can be detected. Nagarajan and colleagues (1999) examined evoked MEG coherence responses in the sensor space of the auditory cortex of adults with poor and good reading abilities. Adults with poor reading abilities showed lower average beta and gamma (20 - $60 \mathrm{~Hz}$ ) coherence than controls. While this study is important in examining coherence activity in dyslexia, it is limited in that it was not imaged in source space and, therefore, anatomical networks could not be explored.

In healthy controls, Kujala and colleagues (2007) identified a left-hemisphere neural network sensitive to reading performance using MEG coherence analysis and imaged source space. Regardless of the stimulus rate, communication within the long-range neural network occurred at a frequency of $8-13 \mathrm{~Hz}$ (alpha). Using a rapid visual presentation task that simulates reading without the need of performing saccades, coherence-based detection of interconnected nodes reproduced several brain regions previously reported to be active in reading. The face motor cortex and the cerebellum, typically associated with speech production, and the orbitofrontal cortex, linked to sensory integration, decision-making and expectation, additionally emerged as densely connected components of the network. The left inferior occipitotemporal cortex, involved in early letter-string or word-specific processing, and the cerebellum were identified as the main forward driving nodes of the network. Interestingly, synchronization within a subset of nodes formed by the left occipitotemporal, the left superior temporal, and orbitofrontal cortex were increased with the subjects' effort to comprehend the text.

Although there is an abundance of evidence supporting the role of phonological processing and awareness in dyslexia, these constructs are insufficient to account for the range of deficits observed in the disorder (Bailey \& Snowling, 2002; Benton, 1975; Kibby, Kroese, Krebbs, Hill, \& Hynd, 2009; Nicolson, Fawcett, Brookes, \& Needle, 2010; Talcott et al., 2000; Zeffiro \& Eden, 2000). Visual attention and working memory affect reading performance and contribute to the processing of phonological information in such a way that deficits in these cognitive domains translate into poor reading performance (Berninger, Raskind, Richards, Abbott, \& Stock, 
2008; Eden, VanMeter, Rumsey, \& Zeffiro, 1996; Facoetti et al., 2010; Pammer, Hansen, Holliday, \& Cornelissen, 2006; Swanson, Howard, \& Saez, 2006; Valdois, Bosse, \& Tainturier, 2004).

Reading proficiency is not only dependent on phonological awareness and on processing, but it also requires the ability to attend, organize, manipulate, and monitor in verbal and visual working memory multiple sequences of sounds and letters (Baddeley, 2007; Bishop \& Snowling, 2004; Conway et al., 2008; Stuss, 2011; Stuss \& Alexander, 2007). Lesions in the left frontal operculum produce selective phonological (pseudoword) processing deficits, suggesting that in addition to the posterior language/reading areas, the left frontal region makes a critical contribution to the phonological processing of words in reading (Fiez, Tranel, Seager Frerichs, \& Damasio, 2006). Some investigators, in fact, have suggested the use of the term phonological short-term memory to emphasize frontal lobe involvement, rather than phonological awareness, as a phenotypic marker for dyslexia for gene linkage studies given its prevalence in this population and clinical validity (Newbury, Bishop, \& Monaco, 2005).

In addition to hypoactivation of left hemispheric posterior regions associated with reading and language, some investigators have demonstrated that individuals with dyslexia show a hyperactivation in the prefrontal gyrus, as well as the caudate and thalamic nuclei, together comprising the fronto-striatal-thalamic system that is essential for working memory (Cropley, Fujita, Innis, \& Nathan, 2006). This hyperactivation of the fronto-striatal-thalamic system may reflect a greater recruitment of working memory resources to support phonological processing, fluency, and word retrieval required in reading (Crosson, 1999; Crosson et al., 2003).

With normal reading development there is a functional shift of activation from fronto-cortical-thalamic systems to temporal-parietal region specialized for language processing needed for reading (Gaillard, Balsamo, Ibrahim, Sachs, \& Xu, 2003). Despite such a relative shift to temporal-parietal regions mediating reading, the functional connection between the inferior parietal cortex and prefrontal cortex remain critical for reading. Using transcranial magnetic stimulation to disrupt the temporal-parietal regions mediating reading, Dong and colleagues (2005) demonstrated that reversible disruption of the left inferior parietal lobule and prefrontal cortex resulted in impaired reading. Dong and colleagues (2005) suggested that attentional (working memory) systems in the prefrontal cortex alert the dorsal language/reading system (inferior parietal lobule, angular gyrus, supramarginal) during the presence of word-like stimuli, which results in top-down activation of the prefrontal cortex and a further amplification of attention of the material being read.

Using a verbal working memory paradigm and independent component analysis of fMRI data, investigators have identified functional pathways that are dysregulated with dyslexia (Wolf et al., 2010). Individuals with dyslexia showed increased functional connectivity within the "phonological" left prefrontal cortex and inferior parietal region and a decreased functional connectivity between the dorsolateral prefrontal cortex and the posterior parietal regions. The latter is a stream that has consistently been implicated in working memory. In addition, the development of functional connectivity between the left and right inferior frontal lobes may, in fact, facilitate treatment response and represent a form of compensatory neuroplastic change in individuals with dyslexia (Farris et al., 2011). Taken together, the evidence suggests that the spatial and verbal working memory systems are intimately involved in the phonological and orthographic processing that underlies reading and are dysregulated in dyslexia.

Despite advances in structural and functional brain imaging, the precise functional neuronal networks that differentiate dyslexics from neurotypical controls are poorly understood. Several attempts have been made to investigate the functional connectivity in the brains of individuals with dyslexia utilizing functional MRI (fMRI) and Positron Emission Tomography (PET); however, these methods lack the temporal resolution to precisely time lock the behavioral elements associated with reading to the hemodynamic or metabolic events measured by these methods. Further, while Diffusion Tensor Imaging (DTI) provides indices of axonal integrity, this imaging technique primarily provides measures of structural rather than functional connectivity.

Reading requires the integration and processing of information from multiple sources and the synchronization of activity from a widely distributed set of brain regions and networks. EEG and MEG methods provide the necessary temporal resolution to investigate the functional and dynamic connectivity that is fundamental to reading. While coherence analysis was first applied to EEG, measuring pairwise correlations of spectral energy in various frequency bands at distinct source sites, these recordings lack the spatial resolution to identify the specific underlying neuronal areas and networks that are activated. MEG coherence source imaging, however, provides the only high temporal and spatial resolution method to simultaneously measure the frequency-dependent, time-locked activity associated with reading and the specific anatomical loci and neural networks comprising 
these dynamic functional connections.

The following study, therefore, investigates the regional brain activation patterns and functional connectivities that differentiate individuals with dyslexia from control subjects using MEG coherence source imaging performing an orthographic or verbal working memory (VWM) task (e.g., letters of the alphabet) and non-orthographic or visual working memory (VWM) task (e.g., shapes).

\section{Methods}

\subsection{Participants}

This study is an analysis of an existing MEG imaging dataset of individuals with dyslexia and age-, gender-, and IQ-matched neurotypical controls evaluated in the Neuromagnetism Laboratory at Henry Ford Hospital, Detroit, MI. A group of men and women with dyslexia $(\mathrm{N}=7$, Males $=5$, Mean Age $=24$, Mean FSIQ = 112) and an age-, gender-, and IQ-matched neurotypical control group $(\mathrm{N}=9$, Males = 8, Mean Age = 26, Mean FSIQ = 115) were recruited from the Michigan Dyslexia Institute and the surrounding metropolitan Detroit Michigan area. Subjects were excluded if they had a history of head injury with loss of consciousness or a comorbid neurological or psychiatric illness or if they were taking or prescribed psychotropic medications within the last three months. Primary language disorders were also ruled out. Normal readers were identified by both academic history and verification by psychometric testing. The dyslexia group consisted of individuals whose performance on word reading and phonological decoding (Wilkinson \& Robertson, 2006, Woodcock, McGrew, \& Mather, 2001) was a minimum of one standard deviation below their scores on standard intelligence testing (Wechsler Abbreviated Scale of Intelligence; Wechsler, 1999) and/or was in the lower $25^{\text {th }}$ percentile for reading (Fletcher, Lyon, Fuchs, \& Barnes, 2007). Eastern Michigan University master's or doctoral students who were blind to the specific hypotheses of the study performed the neuropsychological testing.

\subsection{Study Design}

All participants provided written informed consent prior to entry into the study, and the Institutional Review Boards of Henry Ford Hospital and Eastern Michigan University approved the research protocol prior to initiation of the study and the post-hoc analyses. During MEG imaging, participants were monitored continuously by intercomand video camera. A commercial videotape eraser was used to demagnetizedental work as needed. Three small electrode coils used to locate the subjects' head position with respect to the neuromagnetometer probe were taped to the forehead with two-sided tape. Two additional localization electrodes were taped in front of the subjects' ears on the cheek (just in front of the pre auricular). The subjects then entereda magnetically shielded room to lie comfortably on abed in the supine position. Each subject's head shape was digitized; the locations of fiducial landmarks and the head position electrode coils with respect to the neuromagnetometer detector coils were registered (Fastrack, 4D Neuroimaging, San Diego, CA, USA). The neuromagnetometer helmet containing the detector array was placed around the subject's head in close proximity to the skull surface, and the subject was asked to avoid excessive eye blinks and body movements during data collection. Data collection runs lasted 10 - 15 minutes.

Orthographic (Verbal) and Non-Orthographic (Spatial) Working Memory Paradigms

Subjects' MEG field responses were measured following the visual presentation of a series of upper case letters (verbal or orthographic stimuli) or squares (spatial or non-orthographic stimuli; see Figure 1). Non-orthographic or spatial working memory (SWM) was studied by measuring the subjects' MEG field responses to the visual presentation of a series of white squares presented for 2 seconds every 3 seconds in one of 12 different locations around an imaginary circle (D’Esposito et al., 1998). During each presentation $(\mathrm{N}=40)$, the subjects were asked to mentally determine whether each square was in the same position as the square presented two prior images ago (n-2 back task: Gevins \& Cutillo, 1993). Subjects were instructed to respond only to displays in which this was the case by pushing a keypad with their right forefinger. This test consisted of two trials lasting approximately 7 minutes each. Orthographic or verbal working memory (VWM) was studied by measuring the subjects' MEG field responses to visual presentations of a series of upper case letters for 2 seconds presented every 3 seconds; the position of the block letter did not change with each presentation (D'Esposito et al., 1998). During each presentation $(\mathrm{N}=40)$, the subjects were asked to mentally determine whether the letter being presented 


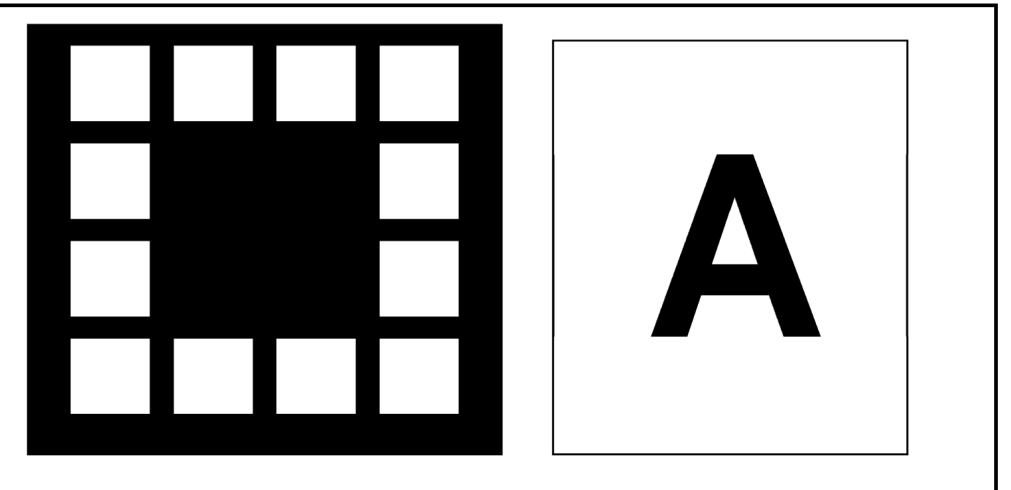

Figure 1. Stimuli used during for the SWM (left) and VWM (right) paradigms, respectively.Stimuli used during for the SWM (top) and VWM (bottom) paradigm. During the SWM paradigm, a series of white squares presented for 2 seconds every 3 seconds in one of 12 different locations around an imaginary circle, while during the VWM paradigm a series of upper case letters for 2 seconds presented every 3 seconds.

was the same as the letter presented two images ago (n-2 back task). Subjects were instructed to respond only to correct targets by pushing a keypad with their right forefinger. This test consisted of two trials lasting approximately 7 minutes each.

The visual stimuli were generated by a Promax Desktop Projector (Model 5950, 1250 Lumens) onto a large mirror tilted 45 degrees to reflect the image upward to another mirror also tilted 45 degrees toward a white screen. The test images on the white screen were viewed from a mirror placed above the subject (the center of the mirror was 14 inches above the face) and tilted 45 degrees to the white screen.

\subsection{MEG Imaging}

The study was performed using a 148-channel neuromagnetometer (4D Neuroimaging WH2500), a helmetshaped device covering the entire adult head, except the face. The individual sensors in the device have SQUID (superconducting quantum interference device) magnetometers, and all measurements were taken inside a magnetically shielded room located in the Neuromagnetism Laboratory at Henry Ford Hospital. During acquisition, the data were band-pass filtered 0.1 to $100 \mathrm{~Hz}$ and digitally sampled and continuously recorded for later analysis. The timing of stimuli was recorded as pulse codes (representing the type of stimulus) on a trigger channel simultaneously collected with the MEG data. In post-processing, noise artifacts due to heart were eliminated using an independent component analysis (ICA) of the data. Singular valued decomposition was used to remove any other artifacts in the data such as mouth movements if needed. Regarding movement artifact, runs were repeated if the coil on head positions exceeds $0.5 \mathrm{~cm}$, although this did not occur during acquisition. As such, we combined the runs before source reconstruction. Regarding noise reduction, 4D Neuroimaging incorporates a set of reference sensors that are used to sample the environmental magnetic fields and create a file that has a set of weights. These weights are subtracted from the data during data collection. Next, the location of events on the trigger and response channels were used to select 2-second epochs of MEG data. These activation epochs were signal averaged and forward and backward band-pass filtered 1.0 to $50 \mathrm{~Hz}$. All epochs had a baseline of $500 \mathrm{~ms}$ before stimuli onset (which is necessary for calculating significance values) and $1500 \mathrm{~ms}$ of data after stimulus onset.

\subsubsection{MRI/MEG Co-Registration}

MEG localizations were computed in reference to the Cartesian coordinate system defined by a set of three anatomical landmarks (fiducial points): the right and left external meatus or pre aurical and the nasion. Prior to the MEG scan, the head surface was digitized using a Polhemus (Fasttrack, 4D Neuroimaging, San Diego, CA, USA). Head digitization points were used to ensure a precise registration, when the points lay on the scalp surface of the MRI scan. STA/R software (4D Neuroimaging, San Diego CA) was used to co-register the MRI row, column, and slice coordinates to the subject's MEG x, y, z co-ordinate system established during data acquisi- 
tion. The techniques for co-registration of MEG and MRI are well established in this laboratory (Bowyer, Mason, Tepley, Smith, \& Barkley, 2003) and allow precise correspondence between anatomical structures and MEG areas of cortical activation with errors less than $5 \mathrm{~mm}$. If the subject's MRI was not available, we used a standard MRI and rescaled to fit the patient's digitized head shape collected during the MEG scan. The standard head and brain model was constructed from the MRI scan of a normal subject, consisting of $124(256 \times 256)$ sagittal T1 images, which includes the entire skin surface of the head.

\subsubsection{MEG Data Analyses}

Source localization of the MEG data was imaged using Multi Resolution-FOCal Underdetermined System Solver (MR-FOCUSS) imaging technique (Moran, Bowyer, \& Tepley, 2005) in MEG-TOOLS, a MatLab based software program (Moran, 2008). A model of the subject's cortical brain was created for the MR-FOCUSS imaging technique. MRI images for each subject were then segmented, and the cortical continuum represented by a cortical model with $\mathrm{x}, \mathrm{y}$, and $\mathrm{z}$ oriented dipoles at approximately 4000 cortical sites were approximated. Cortical sensor sites were distributed such that each represented the same volume of cortical gray matter $\left(\sim 1 \mathrm{~mm}^{3}\right)$. For all MEG techniques, forward model calculations for dipoles utilized a spherical volume conductor model fit to the local curvature of the skull at six locations. The six regions (Frontal, Parietal, and Occipital in both right and left hemispheres) of the skull were fit separately.

The analyses were performed on the averaged MEG evoked epochs from the point of stimulus presentation to $650 \mathrm{~ms}$ after the stimulus onset. Latencies of activation, locations, and normalized amplitudes were determined for cortical sites activated during the subject's performance of the orthographic and non-orthographic working memory tasks. Based on MNI coordinates, average normalized amplitude per active sources in 54 brain regions was calculated. The left and right hemisphere regions of interest (ROIs) were averaged based on total active sources for that ROI to generate normalized mean amplitudes for the ROI over the activation epochs to compare groups. The amplitude normalization procedure allows us to compare across brains by collapsing 4134 brain sources into 54 anatomical areas of interest (Table 1) defined by MNI/Talairach coordinates.

\subsection{MR-FOCUSS}

MR-FOCUSS (Moran et al., 2005) is a current distribution imaging technique that can image simultaneously active regional sources involved in cognitive processing. MR-FOCUSS incorporates the recursive solution approach of FOCUSS as well as control of the $L_{P}$ norm of the solution (focal imaging properties). Thus, MRFOCUSS is able to image both focal and extended sources of brain electric activity. Control of focal imaging properties of the solution and noise suppression is accomplished by the use of an innovative multi-resolution model of source activity. For our cognitive processing studies, MR-FOCUSS solutions were created by averaging a set of 20 solutions at each millisecond. This ensures that the brain activity common to all 20 solutions is in the final image for that millisecond. This technique minimizes initialization bias and allows lower amplitude sources to be more readily imaged. This technique produces a time sequence of whole brain images including both focal and extended source structures for the underlying cortical tissue. Regions with significant activation are determined by a method similar to that used by Sekihara, Nagarajan, Poeppel, Marantz, \& Miyasshita (2001), where the baseline of MEG-imaged brain activity before the stimulus is presented and used to establish a threshold scale of statistical significance for each brain imaging response post stimulus presentation. Image activations were compared between the orthographic (verbal) and non-orthographic (spatial) working memory paradigms for both individuals with dyslexia and control subjects.

\subsection{Coherence Source Imaging Analysis}

For the coherence analysis, the continuous digitally filtered MEG data (band pass 3 - $50 \mathrm{~Hz}$ ) for both the dyslexic and control groups were reloaded into the MEG Tools software (Moran, 2008). For each of the data segments, signals from neuronal sources were isolated using an ICA spatiotemporal decomposition technique designed to extract signals from distinct compact sources that exhibit burst behavior and minimal temporal overlap with other active sources. These ICA signal components have MEG spatial magnetic field patterns corresponding to one or a few spatially distinct compact sources that are much easier to image accurately using the MR-FOCUSS source imaging technique. Separate from the imaging algorithm, the cross-spectrum between ICA signals was calculated. In these cross-spectrum calculations, a sequence of FFT spectra was calculated using $0.5 \mathrm{~s}$ 
Table 1. Left and right hemisphere brain regions analyzed.

\begin{tabular}{c} 
Brain regions \\
Angular gyrus \\
Caudate \\
Cingulate gyrus \\
Cuneus \\
Fusiform gyrus \\
Gyrus rectus \\
Hippocampus \\
Inferior frontal gyrus \\
Inferior occipital gyrus \\
Inferior temporal gyrus \\
Insular cortex \\
Lateral orbitofrontal gyrus \\
Lingual gyrus \\
Middle frontal gyrus \\
Middle occipital gyrus \\
Middle orbitofrontal gyrus \\
Middle temporal gyrus \\
Parahippocampal gyrus \\
Postcentral gyrus \\
Precentral gyrus \\
Precuneus \\
Putamen \\
Superior frontal gyrus \\
Superior occipital gyrus \\
Superior parietal gyrus \\
Superior temporal gyrus \\
Supramarginal gyrus \\
\\
\hline
\end{tabular}

windows and 25\% overlap with FFT amplitudes for 21 frequency bins of 2- $\mathrm{Hz}$ width between 3 and $45 \mathrm{~Hz}$. The imaging results and the signal cross-spectrum were used to calculate the coherence between all pairings of active cortical locations within each of the 21 frequency bins. Finally, for each active source, the average coherence across frequencies and sources was calculated. MEG coherence data from the 21 frequency bins were analyzed as 3 separate frequency ranges ( $3-15 \mathrm{~Hz}, 15-30 \mathrm{~Hz}$, and $30-45 \mathrm{~Hz}$ ) approximating the delta/theta/alpha, beta, and gamma bands and combined to provide an estimate of overall coherence $(3-45 \mathrm{~Hz})$.

Intra-hemispheric coherence was determined by calculating the mean coherence values for each individual hemisphere. Similar connectivity estimates for inter-hemispheric, intra-cortical, and cortical-subcortical pathways were determined by calculating mean coherence values between homotopic regions, within a cortical region, and between cortical and subcortical regions, respectively. The coherence value of each source in a hemisphere or brain area was summed and divided by the number of sources. For details of the coherence calculation, see Elisevich et al., 2011).

\subsection{Statistical Analysis}

Statistical analysis methods used to evaluate the latencies of activation, normalized MEG amplitudes, and coherence data included standard descriptive techniques for continuous variables. To calculate the latencies of MEG activation, pre-determined brain regions were selected based on a manualized region of interest procedure to facilitate analysis of onset of activation and compared using an independent samples $t$-test. An average normalized amplitude per active sources of 54 brain regions (Table 1) was calculated and group differences (individuals 
with dyslexia versus controls) by brain region was determined using an independent samples $t$-test. For both the average normalized amplitude and latency data, statistical significance was set at the $p<.05$ level.

MEG coherence in the cortical sources was calculated for each pair of the 54 brain regions $(N=1431)$ within the theta/alpha (3 - $15 \mathrm{~Hz})$, beta $(15-30 \mathrm{~Hz})$, and low gamma $(30-45 \mathrm{~Hz})$ frequency bands, as well as by combining all three frequency bands to gain an estimate of overall coherence across all frequencies. Coherence values were compared with independent sample $t$-tests using the Benjamini-Hochberg algorithm to control the False Discovery Rate at 0.10 (Benjamini \& Hochberg, 1995). The False Discovery Rate (FDR) is the proportion of tests declared significant that are actually different only due to chance (or the proportion of significant tests that are false positives). The FDR is a widely accepted approach to adjusting for multiple testing in large-scale problems such as the coherence analysis presented here. Such an analysis allows the identification of the anatomical pairs or pathways whose coherence differs between individuals with dyslexia and control subjects that are actually different only due to chance (i.e. false positives).

From each t-test, a z-score was computed according to the method of Efron (2010) to summarize the difference in coherence values between individuals with dyslexia and control subjects in each of the frequency ranges (3 - $15 \mathrm{~Hz}, 15-30 \mathrm{~Hz}$, and $30-45 \mathrm{~Hz}$ ) and their combination (3 - $45 \mathrm{~Hz}$ ). Positive z-scores indicate higher coherence in the dyslexia group. If the null hypothesis is true and there is no difference in coherence between groups, then z-scores will follow a Normal $(0,1)$ distribution. If the distribution of z-scores varies from a Normal distribution, the null hypothesis would not be supported.

Logistic regression analysis was used to evaluate how well MEG coherence activity within specific brain region pairs predicted dyslexia versus control group membership. Statistical significance (Chi-square) was set at the $p<.05$ level. Linear correlational analysis was used to evaluate the relationship between phonological decoding (as measured by Word Attack standard scores of the Woodcock-Johnson III) and MEG coherence activity within specific brain region pairs, with a statistical significance set at the $p<.05$ level.

\section{Results}

Mean latencies of brain activation and averaged normalized amplitudes of activation detected by MEG in the evoked data analysis trials were significantly different between groups and across stimuli. MEG coherence also significantly differed between individuals with dyslexia and matched-controls when performing a working memory paradigm and these results depended on the stimulus presentation (orthographic versus non-orthographic).

\subsection{Latency of MEG Activation}

In contrast to controls, individuals with dyslexia activated frontal cortical regions earlier than controls. The frontal cortical region activation depended on the working memory paradigm.

\subsubsection{Spatial Working Memory (SWM)-Non-Orthographic}

Individuals with dyslexia showed an early mean latency of activation when performing the SWM task in the precentral gyrus (mean latency $=167 \mathrm{~ms}, t(8)=-3.502, p=.008$ ) compared to controls (mean latency = $343 \mathrm{~ms}$; Table 2). In control subjects, MEG activation was initiated in more posterior cortical regions such as the supramarginal gyrus (mean latency $=236 \mathrm{~ms}$ ) and superior temporal gyrus (mean latency $=233 \mathrm{~ms}$ ) during the performance of a SWM task.

\subsubsection{Verbal Working Memory (VWM)-Orthographic}

Individuals with dyslexia showed a significantly early activation in the superior frontal gyrus (mean latency = $209 \mathrm{~ms}, t(12)=-2.021, p=.056)$ during the verbal working memory (VWM) task when compared to controls (mean latency = $325 \mathrm{~ms}$; Table 2). In contrast, control subjects showed an earlier activation in the posterior cortical regions (supramarginal gyrus mean activation latency $=227 \mathrm{~ms}$; superior temporal gyrus mean activation latency $=290 \mathrm{~ms}$ ) prior to engaging fronto-cortical area during the performance of the VMW task.

\subsection{MEG Normalized Amplitudes of Activation}

The pattern and strength of MEG activation differed in individuals with dyslexia and controls, which varied with 
Table 2. Latency of activation in dyslexics performing SWM and VWM tasks.

\begin{tabular}{|c|c|c|c|c|c|}
\hline \multicolumn{6}{|c|}{ Spatial Working Memory (SWM)-non-orthographic } \\
\hline Brain region & Mean (ms) & SD & t-statistic & Degrees of freedom & $p$-value \\
\hline \multicolumn{6}{|l|}{ Precentral gyrus } \\
\hline Control & 343.9 & 109.11 & -3.502 & 8 & $.008^{*}$ \\
\hline Dyslexics & 167.9 & 50.57 & & & \\
\hline \multicolumn{6}{|c|}{ Verbal Working Memory (VWM)-orthographic } \\
\hline Brain region & Mean (ms) & SD & t-statistic & degrees of freedom & $p$-value \\
\hline \multicolumn{6}{|c|}{ Superior frontal gyrus } \\
\hline Control & 325.3 & 149.65 & -2.021 & 12 & $.056^{*}$ \\
\hline Dyslexics & 209.7 & 130.81 & & & \\
\hline
\end{tabular}

${ }^{*} p<.05$.

working memory paradigm and the presentation of orthographic versus non-orthographic stimuli.

\subsubsection{Spatial Working Memory (SWM)-Non-Orthographic}

Of the 54 brain regions examined (Table 1), two regions showed significant differences in MEG activation between dyslexics and controls performing a SWM task. Individuals with dyslexia showed a significantly reduced average normalized amplitude of activation in the right superior temporal gyrus (mean normalized amps $=1.156$ $\mathrm{nAm}, t(13)=2.847, p=.014)$ and right middle temporal gyrus (mean normalized amps $=1.022 \mathrm{nAmp}, t(13)=$ 2.653, $p=.020$ ) compared to controls (Table 3 ). In contrast, control subjects showed a greater mean MEG activation in the right superior temporal gyrus (mean normalized amps $=1.528 \mathrm{nAm}$ ) and right middle temporal gyrus (mean normalized amps $=1.501 \mathrm{nAm}$ ). Figure 2 and Figure 3 compare the MEG normalized amplitudes in the right middle temporal gyrus (Figure 2) and right superior temporal gyrus (Figure 3) in individuals with dyslexia and control subjects.

\subsubsection{Verbal Working Memory (VWM)-Orthographic}

Within the right hemisphere, individuals with dyslexia showed reduced normalized mean amplitudes in the right insular cortex (mean normalized amps $=.569 \mathrm{nAm}, t(13)=2.225, p=.044$ ) and right superior temporal gyrus (mean normalized amps $=1.116 \mathrm{nAm}, t(13)=3.341, p=.005$ ) when performing the VWM compared to controls (Table 3; Figure 4). In contrast to these declines in mean activation, individuals with dyslexia showed an increased mean activation in the right fusiform gyrus during the VWM task compared to controls (mean normalized amps $=1.352 \mathrm{nAm}, t(13)=-2.660, p=.020)$. In the left hemisphere, individuals with dyslexia showed greater mean activation than controls in the left parahippocampal gyrus (mean normalized amps $=.859 \mathrm{nAm}, t$ $(13)=-2.181, p=.048$ ) and left precentral gyrus (mean normalized amps $=1.085 \mathrm{nAm}, t(13)=-2.448, p=.029)$ during a VWM task (Figure 5). Such increases in the right fusiform cortex, left parahippocampal gyrus, and left precentral gyrus in individuals with dyslexia compared to controls seen during the VMW task may represent neuroplastic compensatory changes associated with the disorder.

\subsection{MEG Coherence Source Imaging}

MEG coherence analysis of the cortical sources for each pair of the 54 brain regions $(\mathrm{N}=1431)$ within the theta/alpha (3 - $15 \mathrm{~Hz})$, beta $(15-30 \mathrm{~Hz})$, and low gamma $(30-45 \mathrm{~Hz})$ frequency bands as well as their combination revealed differences between individuals with dyslexia compared to controls that depended on the working memory paradigm and coherence frequency.

\subsubsection{Spatial Working Memory (SWM)-Non-Orthographic}

Individuals with dyslexia demonstrated lower coherent activity when all three frequency ranges were combined (mean $z$ value $=-0.85, t$-stat $=-27.30, p=.000$ ) as well as at the individual low frequency (mean $z$ value $=-0.63$, $t$-stat $=-21.32, p=.000)$, beta (mean $z$ value $=-0.09, t$-stat $=-4.61, p=.00)$, and gamma (mean $z$ value $=-0.99$, 

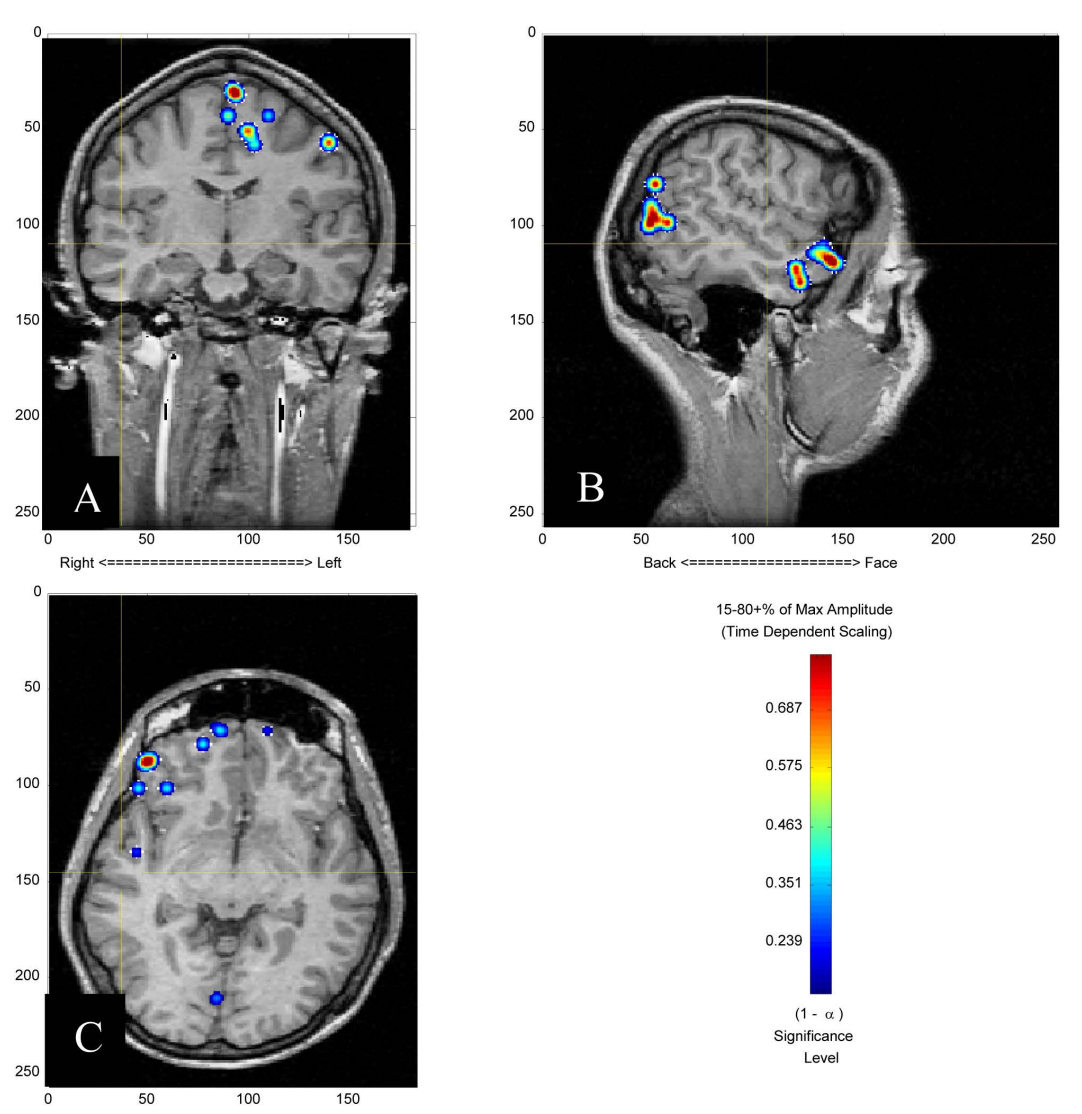

15-80+\% of Max Amplitude
(Time Dependent Scaling)
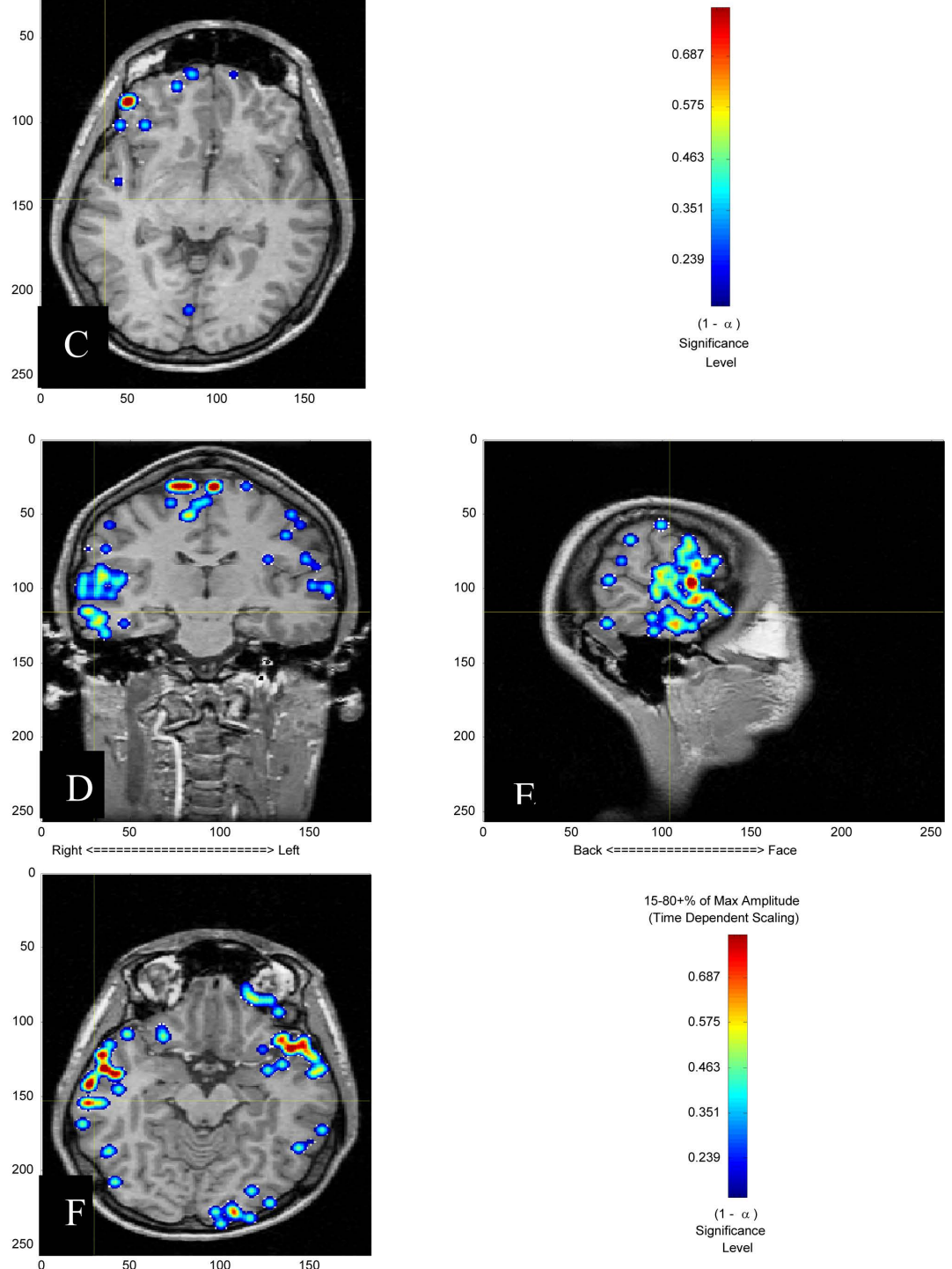

Figure 2. MEG amplitudes in the right middle temporal gyrus during SWM. Coronal, sagittal, and axial planes of MEG normalized amplitudes at the right middle temporal gyrus of dyslexics (A)-(C) and controls (D)-(F) while performing a spatial working memory (SWM) task. 

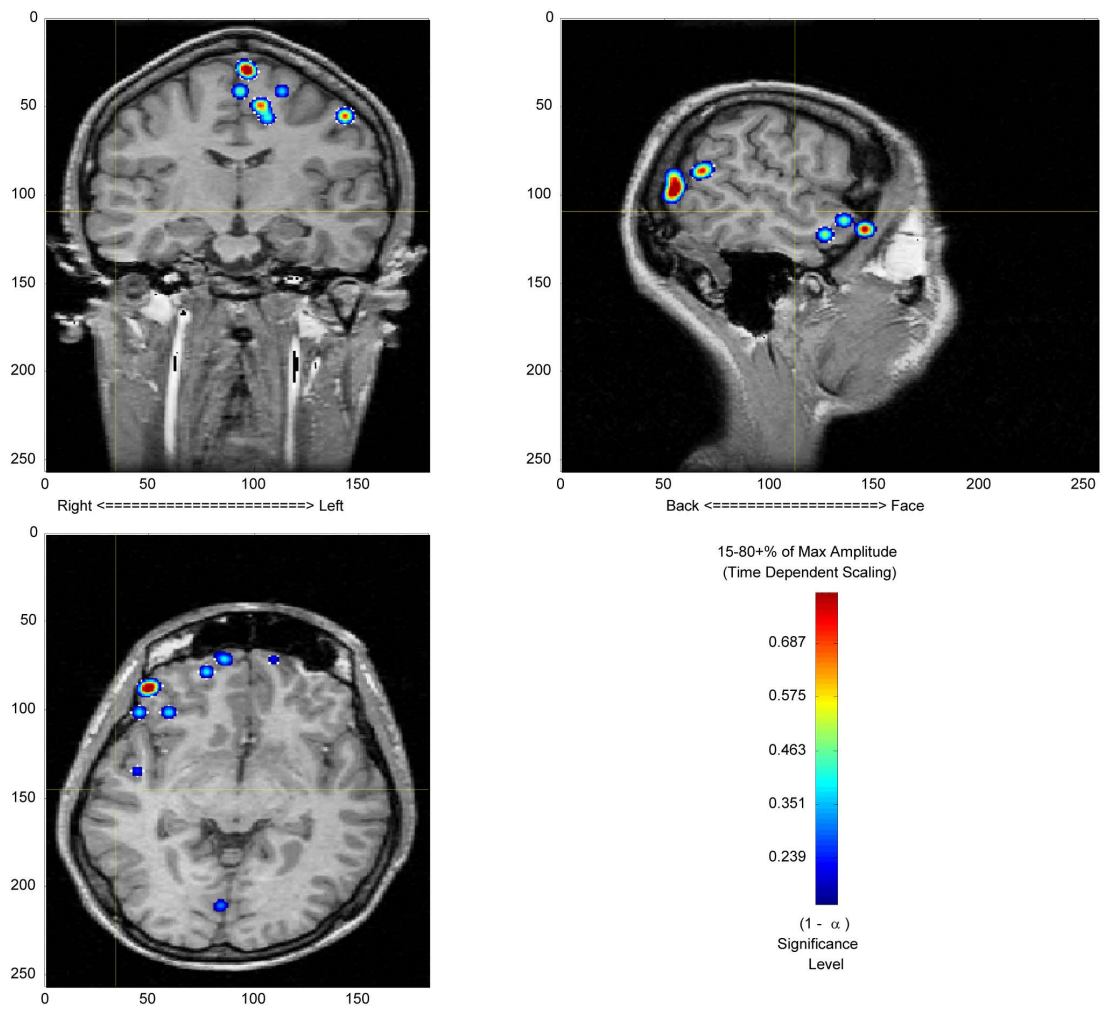

15- $-80+\%$ of Max Amplitude
(Time Dependent Scaling)
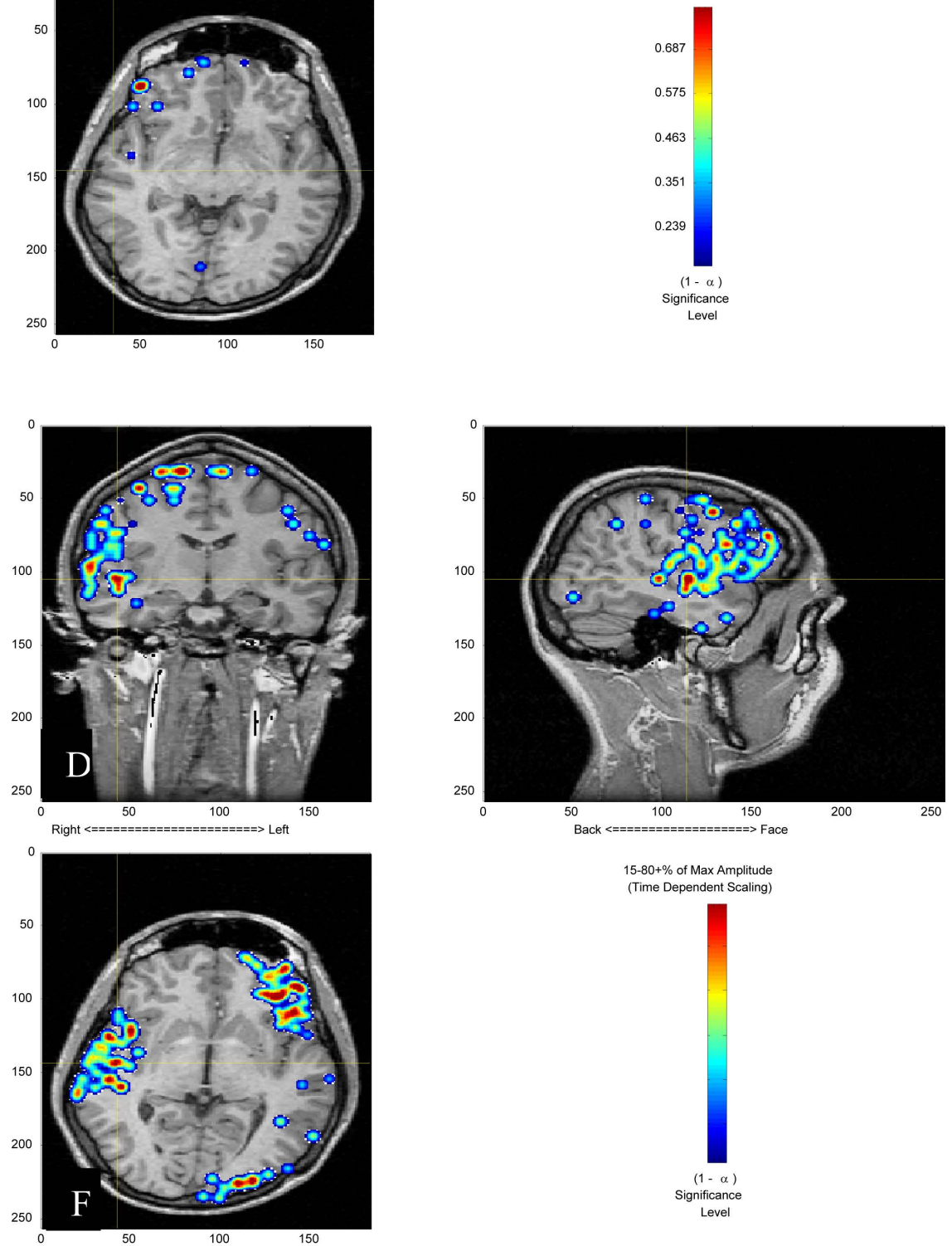

(Time Dependent Scaling)

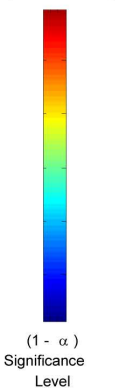

Figure 3. MEG amplitudes in the right superior temporal gyrus during SWM. Coronal, sagittal, and axial planes of MEG normalized amplitudes at the right superior temporal gyrus of dyslexics (A)-(C) and controls (D)-(F) while performing a spatial working memory (SWM) task. 

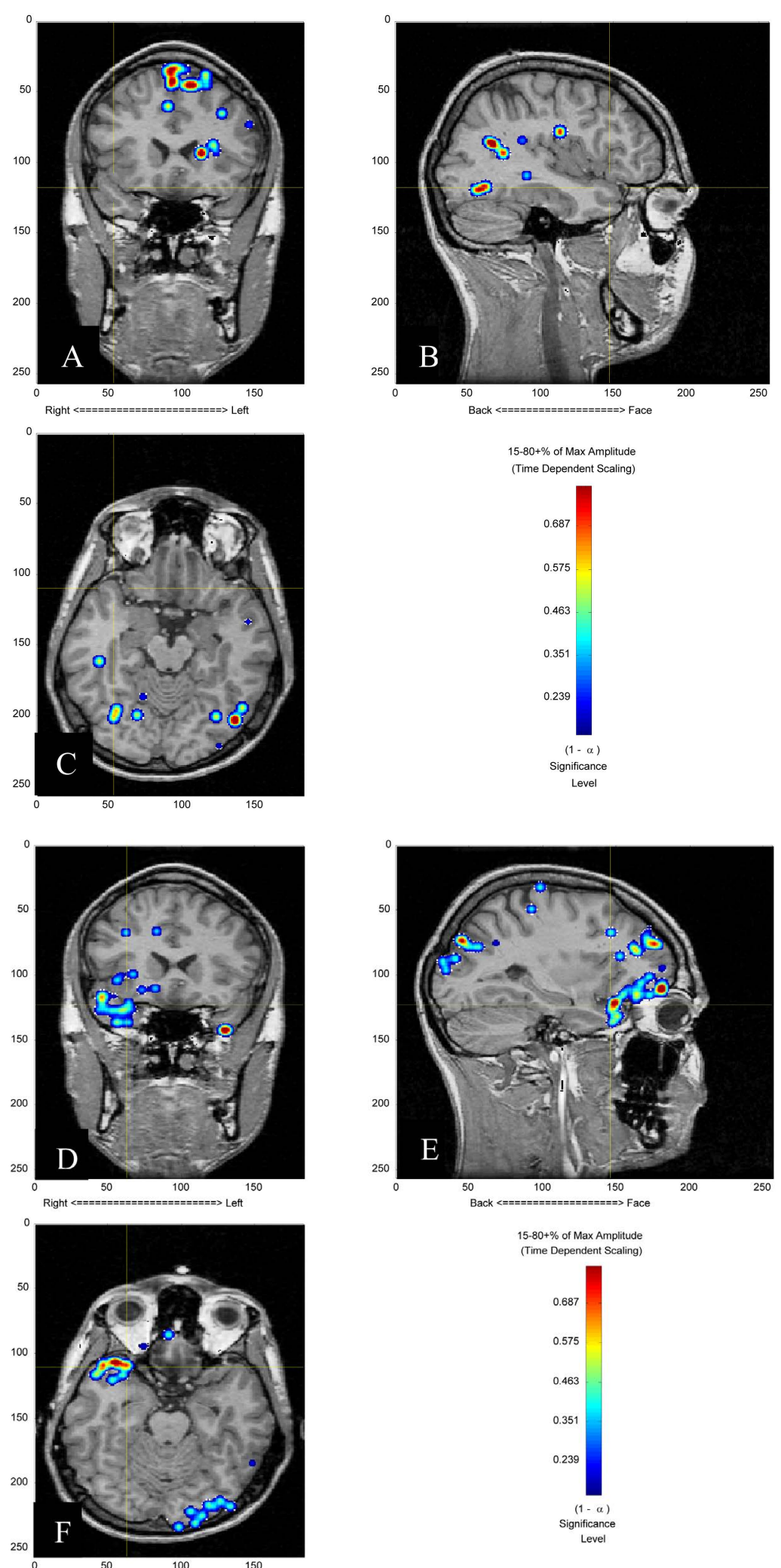

Figure 4. MEG amplitudes in the right superior temporal gyrus during VWM. Coronal, sagittal, and axial planes of MEG normalized amplitudes at the right superior temporal gyrus of dyslexics (A)-(C) and controls (D)-(F) while performing a verbal working memory (VWM) task. 

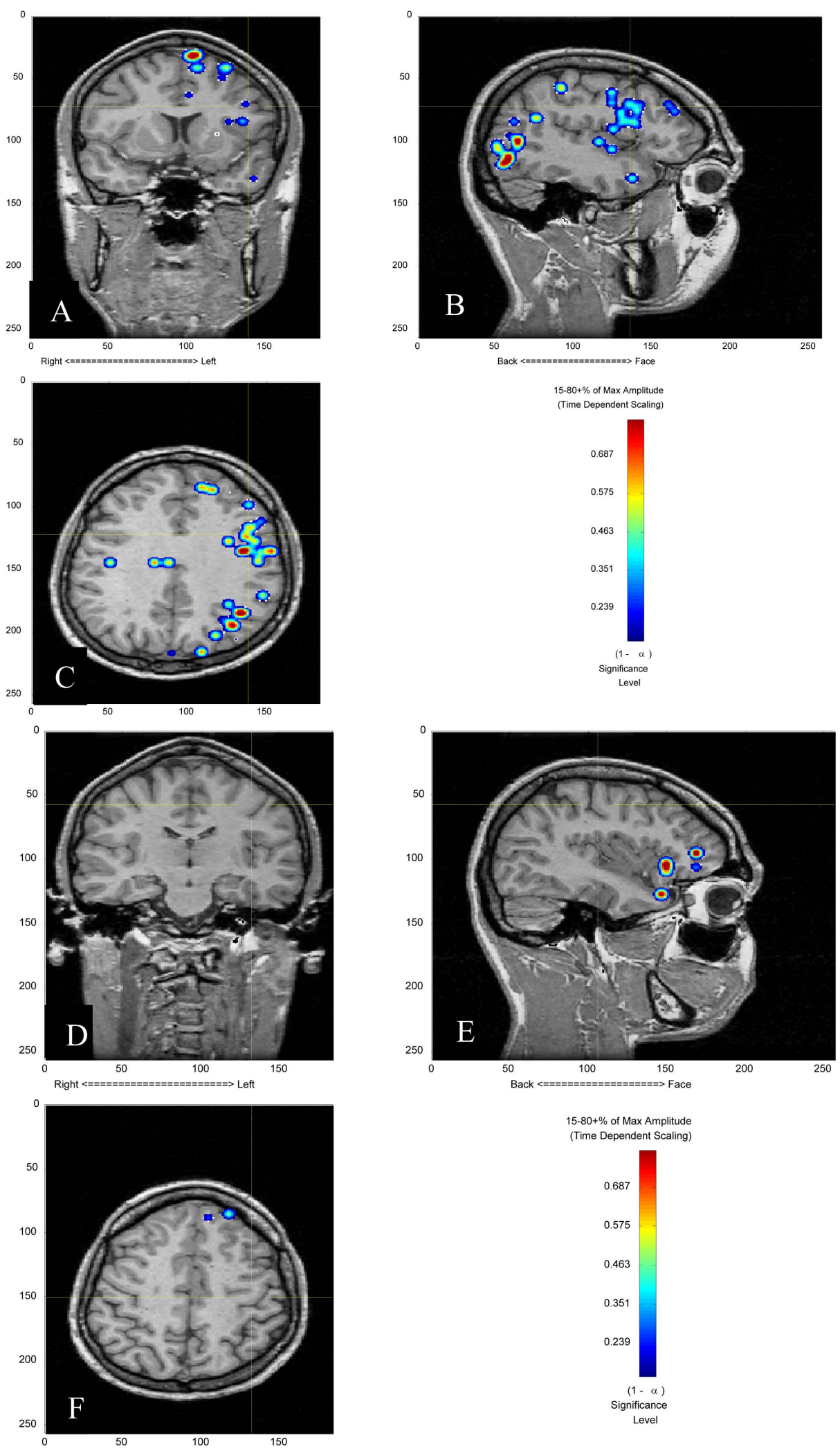

Figure 5. MEG amplitudes in the left precentral gyrus during VWM. Coronal, sagittal, and axial planes of MEG normalized amplitudes in the left precentral gyrus of dyslexics (A)-(C) and controls (D)-(F) while performing a verbal working memory (VWM) task. 
Table 3. Normalized MEG amplitudes in dyslexics performing SWM and VWM tasks.

\begin{tabular}{|c|c|c|c|c|c|}
\hline Brain region & Mean (nAm) & Standard deviation & t-statistic & Degrees of freedom & $p$-value \\
\hline \multicolumn{6}{|c|}{ Spatial Working Memory (SWM)-non-orthographic } \\
\hline \multicolumn{6}{|c|}{ R. middle temporal gyrus } \\
\hline Control & 1.5018 & .36742 & 2.653 & 13 & $.020^{*}$ \\
\hline Dyslexics & 1.0221 & .30007 & & & \\
\hline \multicolumn{6}{|c|}{ R. superior temporal gyrus } \\
\hline Control & 1.5286 & .28093 & 2.847 & 13 & $.014^{*}$ \\
\hline Dyslexics & 1.1562 & .18395 & & & \\
\hline \multicolumn{6}{|c|}{ Verbal Working Memory (VWM)-orthographic } \\
\hline \multicolumn{6}{|c|}{ L. parahippocampal gyrus } \\
\hline Control & .3402 & .20956 & -2.181 & 13 & $.048^{*}$ \\
\hline Dyslexics & .8591 & 63788 & & & \\
\hline \multicolumn{6}{|c|}{ L. precentral gyrus } \\
\hline Control & .8101 & .21209 & -2.448 & 13 & $.029^{*}$ \\
\hline Dyslexics & 1.0850 & .22264 & & & \\
\hline \multicolumn{6}{|c|}{ R. fusiform gyrus } \\
\hline Control & .6171 & .38086 & -2.660 & 13 & $.020^{*}$ \\
\hline Dyslexics & 1.3522 & .66973 & & & \\
\hline \multicolumn{6}{|c|}{ R. insular cortex } \\
\hline Control & .8770 & .27822 & 2.225 & 13 & $.044^{*}$ \\
\hline Dyslexics & .5692 & .25396 & & & \\
\hline \multicolumn{6}{|c|}{ R. superior temporal gyrus } \\
\hline Control & 1.4695 & .16265 & 3.341 & 13 & $.005^{*}$ \\
\hline Dyslexics & 1.1161 & .24416 & & & \\
\hline
\end{tabular}

t-stat $=-40.39, p=.000$ ) frequency bands when performing a spatial working memory task (Table 4). While there was statistically lower MEG coherence at all three frequency ranges, the largest differences were seen at the low and gamma frequency bands. An example of the MEG gamma coherence imaging differences during the SWM, for individuals with dyslexia compared to controls, is displayed in Figure 6.

False Discovery Analysis revealed that of the possible 1431 brain region pairs that were analyzed, 69 region pairs or coherence paths differentiated individuals with dyslexia from controls when the frequency ranges were combined (Table 5). Individuals with dyslexia during SWM showed lower 1) right frontal connectivity, 2) right fronto-temporal connectivity, 3) left and right frontal connectivity, 4) left temporal and right frontal connectivity, and 5) left occipital and right frontal connectivity (Table 6). In contrast, differences in short range connectivity (gamma) in posterior brain regions within the parietal and occipital cortices failed to differentiate the dyslexia and control groups.

Of the 69 brain region pairs that differentiated individuals with dyslexia from control subjects in the SWM task, 41 included the right middle orbitofrontal (23) or the right lateral orbitofrontal (18) as one of the brain region pairs. Of the remaining 28 brain region pairs, 11 included other right frontal regions as one of the pairs: right superior gyrus (4), right inferior gyrus (4), right gyrus rectus (2), and right precentral gyrus (1). Taken together, the MEG coherence results suggested an overall reduced synchronous activity in the right frontal cortex in individuals with dyslexia performing a SWM task, with a convergence of aberrantly lower connectivity particularly in the right middle orbitofrontal gyrus and the right lateral orbitofrontal gyrus for their intra- and inter-hemispheric connections (Figure 6). 

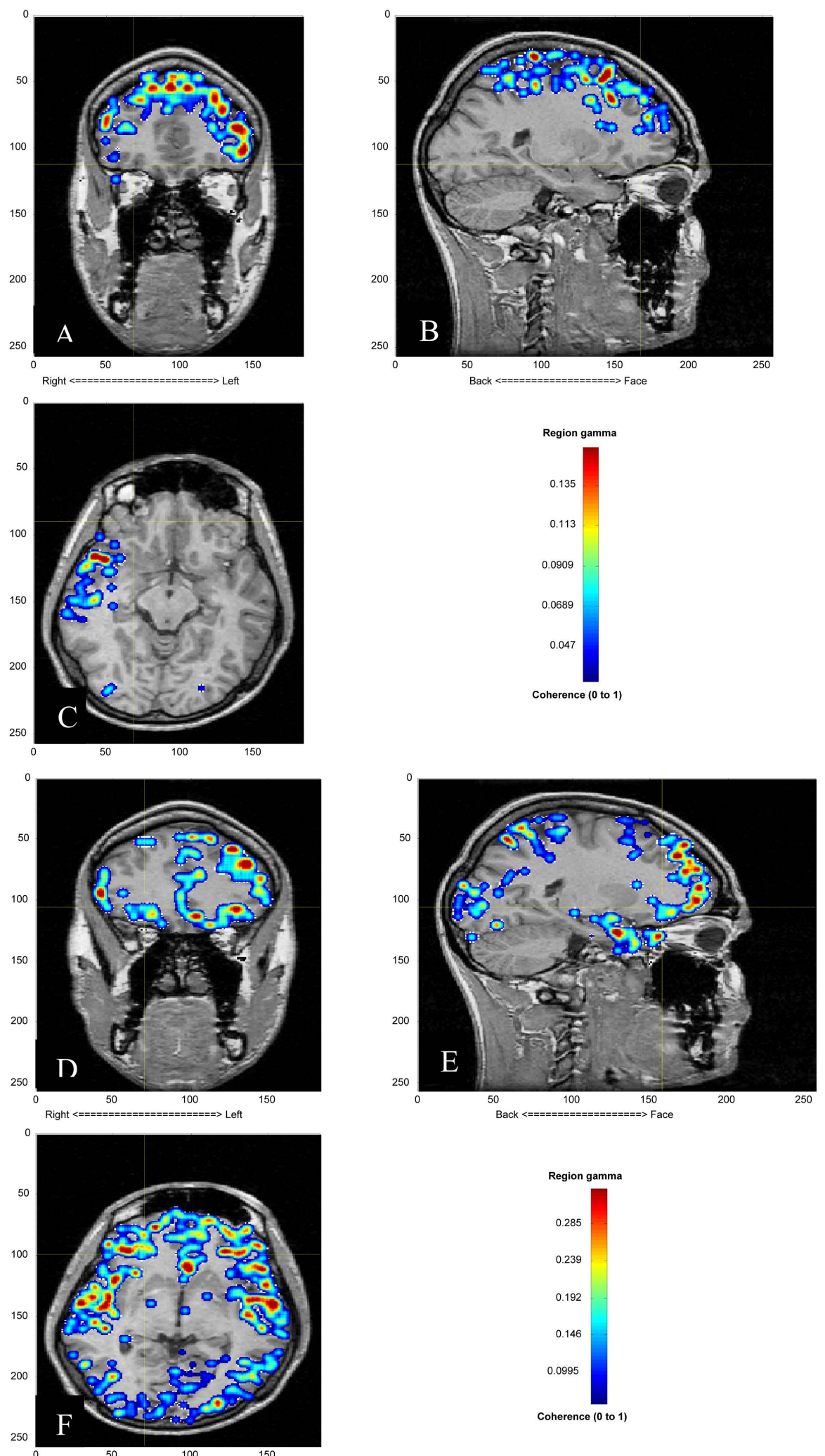

Figure 6. MEG coherence in the right middle and lateral orbitofrontal gyrus during SWM. Coronal, sagittal, and axial planes of MEG coherence (30 - $45 \mathrm{~Hz}$ ) at the right middle and lateral orbitofrontal gyrus of dyslexics (A)-(C) and controls (D)-(F) while performing a spatial working memory (SWM) task. 
Table 4. Differences in MEG coherence distributions in dyslexics versus controls by frequency.

\begin{tabular}{cccccc}
\hline Coherence Frequencies & Mean & Lower Confidence Interval & Upper Confidence Interval & t-Statistic & $p$-value \\
\hline Spatial WM (3 - 15 Hz) & -0.63 & -0.69 & -0.57 & -21.32 & $<0.01$ \\
Spatial WM (15 - 30 Hz) & -0.09 & -0.13 & -0.05 & -4.61 & $<0.01$ \\
Spatial WM (30 - 45 Hz) & -0.99 & -1.03 & -0.94 & -40.39 & $<0.01$ \\
Spatial WM (Overall) & -0.85 & -0.91 & -0.79 & -27.30 & $<0.01$ \\
Verbal WM (3 - 15 Hz) & 0.77 & 0.73 & 0.81 & 35.71 & $<0.01$ \\
Verbal WM (15 - 30 Hz) & 0.08 & 0.03 & 0.14 & 2.92 & $<0.01$ \\
Verbal WM (30 - 45 Hz) & -0.99 & -1.03 & -0.94 & -40.39 & $<0.01$ \\
Verbal WM (Overall) & 0.10 & 0.04 & 0.15 & 3.38 & $<0.01$ \\
\hline
\end{tabular}

Note: WM indicates working memory and overall frequency is a combination of the $3-15 \mathrm{~Hz}, 15$ - $30 \mathrm{~Hz}$, and 30 - $45 \mathrm{~Hz}$ frequency bands.

Logistic regression of the coherence values of the 69 brain region pairs by membership group of individuals with dyslexia versus control subjects was significant (Chi square $=19.036, p<.000, d f=1$ ). Nagelkerke's $\mathrm{R}^{2}$ of .466 indicated a moderately strong relationship between MEG coherence and the prediction of group membership. Overall, predictive success was $84.4 \%: 88.9 \%$ for controls and $77.8 \%$ for individuals with dyslexia. Coherence or connectivity in the right lateral orbitofrontal gyrus and right middle orbitofrontal gyri region pair substantially contributed to group membership (Wald $=13.169, p<.000$ ) such that the forward addition of other pathways failed to significantly add to the predictive value of the model.

Further, there was a significant positive linear correlation between the coherence of the right lateral and right middle orbitofrontal gyri and phonological decoding when all three frequency ranges were assessed $(r=.600, p<.008$, $d f=17)$. This was also true when just the gamma coherence $(30-45 \mathrm{~Hz})$ frequency in this anatomical pathway was correlated with phonological decoding abilities $(r=.796, p<.032, d f=6)$.

\subsubsection{Verbal Working Memory (VWM)-Orthographic}

Individuals with dyslexia demonstrated an overall modestly greater synchronization when power spectra in all three frequency ranges were combined (mean $z$ value $=0.10, t$-stat $=3.38, p=.00$ ) while performing a verbal memory task (Table 4). Analysis of the MEG coherence in individual frequency ranges revealed that individuals with dyslexia showed a higher coherence at the low(mean $z$ value $=0.77, t$-stat $=35.71, p=.000$ ) and beta (mean $z$ value $=0.08, t$-stat $=2.92, p=.00$ ) frequency bands but lower coherence at the gamma frequency band (mean $z$ value $=-0.99$, $t$-stat $=-40.39, p=.000$ ) when performing a verbal working memory task (Table 4). While there was statistically higher coherent activity in both the low and beta frequency ranges, the largest differences were seen at the low frequency band, with only a modestly higher coherence in the beta $(15-30 \mathrm{~Hz})$ frequency range.

False Discovery Analysis failed to identify which of the 1431 brain region pairs differentiated individuals with dyslexia and control subjects when performing a verbal working memory task when all three frequencies were combined ( 3 - $45 \mathrm{~Hz}$ ). This was in part due to the opposing differences in coherence seen in the low (3 - 15 $\mathrm{Hz}$ ) and gamma (30 - $45 \mathrm{~Hz}$ ) frequency ranges. When the False Discovery Analysis was applied separately for each of the three frequency ranges, there was insufficient statistical power to reliably identify specific brain region pairs or paths that reliably differentiated dyslexics from controls during VWM.

\section{Discussion}

The findings of this study provide the first comprehensive view of the brain regions and functional neural circuits that are differentially active in individuals with dyslexia and control subjects during the performance of verbal and spatial working memory tasks, significantly advancing our understanding of the pathophysiology of this disorder. Not only did individuals with dyslexia process orthographic information, as letters, differently than controls, but the two groups also differed in their processing of spatial information in the context of working memory, further highlighting the importance of visual working memory in the etiology of this disorder. In fact, synchronous oscillatory activity in the right middle and right lateral orbitofrontal gyri during the performance of 
Table 5. Differential coherences in dyslexics performing a spatial working memory task.

\begin{tabular}{|c|c|c|}
\hline Coherence pathways & z-value & $p$-value \\
\hline R. lateral orbitofrontal gyrus-R. middle orbitofrontal gyrus & -4.33 & $<0.0000$ \\
\hline L. superior temporal gyrus-R. middle orbitofrontal gyrus & -4.22 & $<0.0000$ \\
\hline R. middle orbitofrontal gyrus-R. middle temporal gyrus & -4.15 & $<0.0000$ \\
\hline L. superior temporal gyrus-R. lateral orbitofrontal gyrus & -4.20 & $<0.0000$ \\
\hline L. middle temporal gyrus-R. middle orbitofrontal gyrus & -4.15 & $<0.0000$ \\
\hline R. lateral orbitofrontal gyrus-R. superior frontal gyrus & -4.12 & $<0.0000$ \\
\hline L. inferior occipital gyrus-R. middle orbitofrontal gyrus & -4.04 & 0.0001 \\
\hline L. middle temporal gyrus-R. lateral orbitofrontal gyrus & -4.14 & 0.0001 \\
\hline R. middle orbitofrontal gyrus-R. superior temporal gyrus & -3.96 & 0.0001 \\
\hline L. gyrus rectus-R. lateral orbitofrontal gyrus & -3.91 & 0.0001 \\
\hline R. lateral orbitofrontal gyrus-R. superior temporal gyrus & -3.96 & 0.0002 \\
\hline R. middle orbitofrontal gyrus-R. superior frontal gyrus & -3.78 & 0.0002 \\
\hline L. lateral orbitofrontal gyrus-R. middle orbitofrontal gyrus & -3.80 & 0.0002 \\
\hline L. superior frontal gyrus-R. lateral orbitofrontal gyrus & -3.84 & 0.0002 \\
\hline R. gyrus rectus-R. lateral orbitofrontal gyrus & -3.67 & 0.0002 \\
\hline L. gyrus rectus-R. middle orbitofrontal gyrus & -3.68 & 0.0002 \\
\hline L. gyrus rectus-L. middle orbitofrontal gyrus & -3.62 & 0.0003 \\
\hline L. middle orbitofrontal gyrus-R. middle orbitofrontal gyrus & -3.67 & 0.0003 \\
\hline L. middle orbitofrontal gyrus-R. lateral orbitofrontal gyrus & -3.68 & 0.0003 \\
\hline L. superior frontal gyrus-R. middle orbitofrontal gyrus & -3.58 & 0.0004 \\
\hline L. inferior temporal gyrus-R. middle orbitofrontal gyrus & -3.53 & 0.0004 \\
\hline L. middle occipital gyrus-R. lateral orbitofrontal gyrus & -3.55 & 0.0005 \\
\hline R. lateral orbitofrontal gyrus-R. middle temporal gyrus & -3.58 & 0.0005 \\
\hline L. gyrus rectus-R. inferior frontal gyrus & -3.46 & 0.0005 \\
\hline L. inferior occipital gyrus-R. lateral orbitofrontal gyrus & -3.44 & 0.0007 \\
\hline R. inferior temporal gyrus-R. lateral orbitofrontal gyrus & -3.48 & 0.0007 \\
\hline L. fusiform gyrus-R. lateral orbitofrontal gyrus & -3.42 & 0.0007 \\
\hline R. inferior temporal gyrus-R. middle orbitofrontal gyrus & -3.42 & 0.0007 \\
\hline L. inferior temporal gyrus-R. lateral orbitofrontal gyrus & -3.45 & 0.0007 \\
\hline L. superior temporal gyrus-R. superior temporal gyrus & -3.37 & 0.0009 \\
\hline L. middle occipital gyrus-R. middle orbitofrontal gyrus & -3.32 & 0.0009 \\
\hline L. fusiform gyrus-R. middle orbitofrontal gyrus & -3.34 & 0.0010 \\
\hline L. gyrus rectus-R. superior temporal gyrus & -3.30 & 0.0010 \\
\hline R. inferior frontal gyrus-R. middle orbitofrontal gyrus & -3.37 & 0.0011 \\
\hline R. gyrus rectus-R. middle orbitofrontal gyrus & -3.26 & 0.0011 \\
\hline L. gyrus rectus-L. superior temporal gyrus & -3.25 & 0.0012 \\
\hline R. fusiform gyrus-R. lateral orbitofrontal gyrus & -3.24 & 0.0013 \\
\hline R. gyrus rectus-R. inferior frontal gyrus & -3.22 & 0.0013 \\
\hline
\end{tabular}




\section{Continued}
L. gyrus rectus-R. middle temporal gyrus
L. gyrus rectus-R. inferior temporal gyrus

R. inferior frontal gyrus-R. lateral orbitofrontal gyrus

L. precentral gyrus-R. middle orbitofrontal gyrus

L. gyrus rectus-L. lateral orbitofrontal gyrus

L. gyrus rectus-R. fusiform gyrus

L. middle occipital gyrus-R. superior frontal gyrus

L. gyrus rectus-R. superior frontal gyrus

R. fusiform gyrus-R. middle orbitofrontal gyrus

L. gyrus rectus-L. middle frontal gyrus

L. middle temporal gyrus-R. superior temporal gyrus

L. superior temporal gyrus-R. superior frontal gyrus

L. gyrus rectus-L. superior frontal gyrus

L. middle temporal gyrus-R. superior frontal gyrus

L. parahippocampal gyrus-R. middle orbitofrontal gyrus

R. inferior occipital gyrus-R. middle orbitofrontal gyrus

L. gyrus rectus-R. precentral gyrus

L. fusiform gyrus-L. gyrus rectus

L. middle orbitofrontal gyrus-L. superior temporal gyrus

L. middle occipital gyrus-L. middle temporal gyrus

R. gyrus rectus-R. superior temporal gyrus

L. middle temporal gyrus-R. inferior frontal gyrus

L.gyrus rectus-L. middle temporal gyrus

R. middle orbitofrontal gyrus-R. precentral gyrus

L. middle occipital gyrus-R. superior temporal gyrus

L. middle frontal gyrus-R. lateral orbitofrontal gyrus

L. middle occipital gyrus-L. superior temporal gyrus

L. superior temporal gyrus-R. inferior frontal gyrus

L. middle orbitofrontal gyrus-L. middle temporal gyrus

L. middle orbitofrontal gyrus-R. gyrus rectus

\begin{tabular}{ll}
-3.21 & 0.0014 \\
-3.15 & 0.0017 \\
-3.30 & 0.0018 \\
-3.15 & 0.0018 \\
-3.11 & 0.0019 \\
-3.15 & 0.0019 \\
-3.09 & 0.0020 \\
-3.07 & 0.0021 \\
-3.08 & 0.0023 \\
-3.05 & 0.0023 \\
-3.07 & 0.0027 \\
-3.02 & 0.0028 \\
-2.98 & 0.0029 \\
-3.01 & 0.0029 \\
-3.01 & 0.0030 \\
-2.98 & 0.0030 \\
-2.95 & 0.0032 \\
-2.95 & 0.0032 \\
-2.95 & 0.0035 \\
-2.95 & 0.0035 \\
-2.92 & 0.0036 \\
-3.01 & 0.0036 \\
-2.92 & 0.0036 \\
-2.95 & 0.0038 \\
-2.91 & 0.0039 \\
-2.93 & 0.0039 \\
-2.90 & 0.0040 \\
-2.96 & 0.0041 \\
-2.84 & 0.0046 \\
\hline & \\
\hline & \\
\hline &
\end{tabular}

0.0014

.0017

0018

0.0019

0.0020

0.0021

0.0023

0.0023

0.0027

0.0028

0.0029

0.0030

0.0030

0.0032

0.0032

0035

0.0035

0.0036

0036

0.0036

0.0038

.0039

0.0039

0.0040

.0041

0.0046

Note: Differential coherences between brain region pairs that distinguish dyslexics from controls performing a spatial working memory task presented in descending order of significance ( $p$-values). L. signifies left hemispheric structures and R. signifies right hemispheric structures.

the spatial working memory task was sufficiently robust to predict group membership (dyslexia versus controls) at an overall rate of $84.4 \%$ and was positively correlated to phonological ability.

In the present study, MEG neuroimaging during the performance of orthographic and non-orthographic working memory tasks suggests fronto-temporal inefficiencies/impairments in individuals with dyslexia as evidenced by the early onset and reliance on prefrontal cortical areas, the differential activation of fronto-temporal brain systems, and an altered pattern of functional connectivity in the fronto-temporal pathways mediating these behaviors.

\subsection{Latency}

In contrast to controls, individuals with dyslexia tended to recruit prefrontal cortical regions earlier over more 
Table 6. Differential coherence pathways during spatial working memory in dyslexics.

Intra-hemispheric (associational)

Frontal-frontal (right)

R. lateral orbitofrontal gyrus-R. middle orbitofrontal gyrus

R. lateral orbitofrontal gyrus-R. superior frontal gyrus

R. middle orbitofrontal gyrus-R. superior frontal gyrus

R. gyrus rectus-R. lateral orbitofrontal gyrus

R. inferior frontal gyrus-R. middle orbitofrontal gyrus

$R$. gyrus rectus-R. middle orbitofrontal gyrus

R. gyrus rectus-R. inferior frontal gyrus

R. inferior frontal gyrus-R. lateral orbitofrontal gyrus

R. middle orbitofrontal gyrus-R. precentral gyrus

Frontal-Frontal (left)

L. gyrus rectus-L. middle orbitofrontal gyrus

L. gyrus rectus-L. lateral orbitofrontal gyrus

L. gyrus rectus-L. middle frontal gyrus

L. gyrus rectus-L. superior frontal gyrus

Frontal-Temporal (right)

R. middle orbitofrontal gyrus-R. middle temporal gyrus

R. middle orbitofrontal gyrus-R. superior temporal gyrus

R. lateral orbitofrontal gyrus-R. superior temporal gyrus

R. lateral orbitofrontal gyrus-R. middle temporal gyrus

R. inferior temporal gyrus-R. lateral orbitofrontal gyrus

R. inferior temporal gyrus-R. middle orbitofrontal gyrus

R. fusiform gyrus-R. lateral orbitofrontal gyrus

R. fusiform gyrus-R. middle orbitofrontal gyrus

R.gyrus rectus-R.superior temporal gyrus

Frontal-Temporal (left)

L. gyrus rectus-L. superior temporal gyrus

L. fusiform gyrus-L. gyrus rectus

L. middle orbitofrontal gyrus-L. superior temporal gyrus

L. gyrus rectus-L. middle temporal gyrus

L. middle orbitofrontal gyrus-L. middle temporal gyrus

Occipital-Frontal (right)

R. inferior occipital gyrus-R. middle orbitofrontal gyrus

Posterior Local (right)

None

Posterior Local (left)

L. middle occipital gyrus-L. middle temporal gyrus

L. middle occipital gyrus-L. superior temporal gyrus

Inter (Cross)-Hemispheric 


\section{Continued}

Frontal-Frontal

L. gyrus rectus-R. lateral orbitofrontal gyrus

L. lateral orbitofrontal gyrus-R. middle orbitofrontal gyrus

L. superior frontal gyrus-R. lateral orbitofrontal gyrus

L. gyrus rectus-R. middle orbitofrontal gyrus

L. middle orbitofrontal gyrus-R. lateral orbitofrontal gyrus

L. superior frontal gyrus-R. middle orbitofrontal gyrus

L. gyrus rectus-R. inferior frontal gyrus

L. precentral gyrus-R. middle orbitofrontal gyrus

L. middle frontal gyrus-R. lateral orbitofrontal gyrus

L. middle orbitofrontal gyrus-R. gyrus rectus

L. gyrus rectus-R. superior frontal gyrus

L. middle orbitofrontal gyrus-R. middle orbitofrontal gyrus

L. lateral orbitofrontal gyrus-R. lateral orbitofrontal gyrus

Temporal-Frontal

L. superior temporal gyrus-R. middle orbitofrontal gyrus

L. superior temporal gyrus-R. lateral orbitofrontal gyrus

L. middle temporal gyrus-R. middle orbitofrontal gyrus

L. middle temporal gyrus-R. lateral orbitofrontal gyrus

L. inferior temporal gyrus-R. middle orbitofrontal gyrus

L. fusiform gyrus-R. lateral orbitofrontal gyrus

L. inferior temporal gyrus-R. lateral orbitofrontal gyrus

L. fusiform gyrus-R. middle orbitofrontal gyrus

L. superior temporal gyrus-R. superior frontal gyrus

L. middle temporal gyrus-R. superior frontal gyrus

L. parahippocampal gyrus-R. middle orbitofrontal gyrus

L. middle temporal gyrus-R. inferior frontal gyrus

L. superior temporal gyrus-R. inferior frontal gyrus

L. gyrus rectus-R. middle temporal gyrus

Occipital-Frontal

L. middle occipital gyrus-R. lateral orbitofrontal gyrus

L. inferior occipital gyrus-R. lateral orbitofrontal gyrus

L. middle occipital gyrus-R. middle orbitofrontal gyrus

L. middle occipital gyrus-R. superior frontal gyrus

Temporal-Temporal

L. middle temporal gyrus-R. superior temporal gyrus

L. sperio temporal gyrus-R. superior temporal gyrus

Occipital-Temporal

L. middle occipital gyrus-R. superior temporal gyrus

Note: Differential coherences between brain region pairs that distinguish individuals with dyslexics from controls performing a spatial working memory task. L. signifies left hemispheric structures and R. signifies right hemispheric structures. 
posterior temporal or parietal cortical gyri as the superior temporal gyrus and the supramarginal gyrus, regardless of whether they were processing orthographic or non-orthographic information. Specifically, individuals with dyslexia showed an early activation in the superior frontal gyrus (209 ms), whereas control subjects showed an earlier activation in the language-related posterior cortical regions (supramarginal gyrus $=227 \mathrm{~ms}$; superior temporal gyrus $=290 \mathrm{~ms}$ ), prior to engaging superior frontal gyrus $(325 \mathrm{~ms})$ during the performance of the verbal working memory task. Similarly, during the spatial working task, individuals with dyslexia showed an early latency of activation in the precentral gyrus (167 ms) compared to controls (343 ms), who initiated in more posterior cortical regions of the supramarginal gyrus $(236 \mathrm{~ms}$ ) and superior temporal gyrus (233 ms) during the SWM task.

These findings in individuals with dyslexia performing verbal and spatial working memory tasks are consistent with a general reduced reliance or dysregulation on temporo-parietal circuits in favor of fronto-cortical pathways or from more specialized regions mediating functions such as language and multi-modal attention and phonological awareness, compared to those mediating goal-directed, executive, attentional, monitoring, and manipulative functions (Corina et al., 2001; Hoeft et al., 2007; Mc Candliss \& Noble, 2003; Richlan, 2012; Shaywitz et al., 2004; Shaywitz et al., 1998; Temple, 2002). Activation of the left superior temporal and parietal cortex in individuals with dyslexia is typically weaker, delayed, and less sensitive to the orthographic and phonemic content of the stimulus than controls (Helenius, Tarkiainen, Cornelissen, Hansen, \& Salmelin, 1999; Laine, Salmelin, Helenius, \& Marttila, 2000; Simos, Breier, Zouridakis, \& Papanicolaou, 1998) with a suggestive, though inconsistent, compensatory recruitment of the left inferior frontal, right inferior frontal, or bilateral fronto-cortical areas in response to letters and words (Richlan, Kronbichler, \& Wimmer, 2009). In the present study, individuals with dyslexia demonstrated a comparatively early activation and reliance on the precentral gyrus and the superior frontal gyrus during the SWM and VWM paradigms, respectively. These fronto-cortical regions are involved in higher levels of working memory processing (monitoring and manipulation) that remain oriented to spatial and verbal input (Boisgueheneuc et al., 2006; Machizawa, Kanai, Rees, \& Driver, 2010), and the early activation in these brain areas in individuals with dyslexia suggest a differential processing of orthographic and non-orthographic information compared to controls.

\subsection{MEG Activation Patterns}

The pattern of MEG activation differed in individuals with dyslexia and controls, which varied with the working memory paradigm and the presentation of orthographic versus non-orthographic stimuli. Of 54 brain regions examined in the left and right hemisphere, individuals with dyslexia demonstrated reduced MEG activation in the right superior and right middle temporal gyri compared to neurotypical controls during the spatial working memory task. Reduced activation in the right superior gyrus may reflect a primary deficit in dyslexia, as the rostral part of the superior temporal gyrus acts as an interface between the dorsal and ventral streams of visual input processing to allow the exploration of both object-related and space-related information (Karnath, 2001), which is likely to be integral in reading.

The activational pattern and differences between individuals with dyslexia and controls are more complex with the presentation of orthographic stimuli (i.e. letters) and may reflect a combination of primary deficits and compensatory changes. As seen with the spatial working memory task, individuals with dyslexia showed a reduced activation in the right superior temporal gyrus, suggestive of an orthographic-independent processing deficit in the dorsal and ventral streams. During the orthographic working memory task, individuals also showed a reduction in mean activation in the right insular cortex compared to controls, suggestive of a deficiency in switching between brain networks and stimulus modalities that has been associated with this brain region (Sridharan, Levitin, \& Menon, 2008).

In contrast to these declines in mean MEG activation, individuals with dyslexia showed increases in activation in the right fusiform gyrus, left parahippocampal gyrus, and left precentral gyrus during the VWM task, which may represent neuroplastic compensatory changes associated with the disorder. In general, during the VWM task, dyslexics showed a greater reliance on and activation of the right hemisphere, particularly the fusiform gyrus, and left hemispheric non-specialized language-related regions of the parahippocampal and precentral gyri, which are implicated in memory formation and higher level working memory processing, respectively. Concurrent with these presumed compensatory increases in activation were reductions in right hemispheric regions, critical for the integration of object- and space-related processing streams (right superior temporal gyrus) 
and in switching between brain networks and stimulus modalities (right insular cortex).

\subsection{MEG Coherence Frequency Ranges}

MEG coherence analysis of the 54 brain region pairs within each of the three frequency bands, as well as their combination, revealed marked differences between individuals with dyslexia and controls that depended on the working memory paradigm and coherence frequency band. During a SWM paradigm, individuals with dyslexia demonstrated an overall lower coherence activity when all three coherence frequency ranges were combined and when frequency was analyzed separately, with the largest differences seen at the theta/alpha and gamma frequency bands. In contrast, during the VWM paradigm, individuals with dyslexia demonstrated an overall modestly greater coherence activity when all three coherence frequency ranges were combined. Analysis of the MEG coherences at separate frequency ranges during VWM revealed that individuals with dyslexia showed higher coherence at the theta/alpha and beta frequency bands but lower coherence in the gamma frequency band. While there was statistically higher coherence activity in the two lower frequency ranges, the largest differences were in the low $3-15 \mathrm{~Hz}$ frequency range. Unlike the coherence results seen during the spatial working memory task that demonstrated a consistent decline in coherent activity at all the frequency ranges, during the verbal working memory task individuals with dyslexia showed an increased MEG coherence at the low frequency range, suggestive of a compensatory change in connectivity, and a concomitant decline in the high frequency range. As low EEG coherence frequencies (e.g., 1 - $10 \mathrm{~Hz}$ ) reflect non-language specific components of word processing such as the sensory, attentional, and mnemonic parts of the task and higher gamma frequencies reflect higher-order orthographic cognitive processing (Weiss \& Mueller, 2003), the increased coherence activity observed here in the low frequency range suggests that in dyslexia there is a shift or greater reliance on attentional fronto-cortical systems from those left parietal-temporal and occipito-temporal higher-order orthographic cognitive processing systems.

Support for this hypothesis comes from an EEG coherence study (Arns, Peters, Breteler, \& Verhoeven, 2007) that reported a symmetric increase in coherence for the lower frequency bands (delta and theta) in frontal and left temporal regions and a specific right-temporocentral increase in coherence for the higher frequency bands (alpha and beta). Significant correlations were observed between subtests such as Rapid Naming Letters, Articulation, Spelling and Phoneme Deletion, and the EEG coherence profiles.

\subsection{MEG Connectivity Pathways and Neuroanatomical Tracts}

The overall reduction in synchronous oscillatory activity in the present study in individuals with dyslexia performing a spatial working memory task reflected lower 1) right frontal connectivity, 2) right fronto-temporal connectivity, 3) left and right frontal connectivity, 4) left temporal and right frontal connectivity, and 5) left occipital and right frontal connectivity. Homotopic commissural connectivity that differentiated individuals with dyslexia from neurotypical controls was limited to fronto-temporal projections (medial orbitofrontal, lateral orbitofrontal, and superior temporal gyrus). In contrast, differences in short range connectivity in posterior brain regions within the parietal and occipital cortices failed to differentiate individuals with dyslexia and control subjects.

Analysis of the functional pathways that demonstrate a significantly reduced coherence activity in individuals with dyslexia when performing a spatial working memory task suggests a dysregulation and diminished connectivity in both intra- and inter-hemispheric pathways, with the crossed inter-hemispheric pathways being predominant. Such reduced inter-hemispheric connectivity in individuals with dyslexia is likely related to morphological differences seen in the callosal fibers of dyslexic readers in the mid-body/isthmus regions that contains inter-hemispheric fibers from primary and secondary auditory cortices (Fine, Semrud-Clikeman, Keith, Stapleton, \& Hynd, 2007; Hasan et al., 2012; Robichon, Bouchard, Demonet, \& Habib, 2000; von Plessen et al., 2002) and the genu of the corpus callosum that connects the frontal lobes (Hynd et al., 1995).

Intra-hemispherically, there is reduced connectivity in local cortical areas, particularly in the right and left frontal cortex, as well as in long bilateral connections that extend from the temporal to frontal cortex and, to a limited extent, from the occipital to frontal cortex. Consistent with these differences in functional connectivity, DTI studies suggest that the brains of individuals with dyslexia have reduced FA and coherence bilaterally in the frontal-temporal pathway (superior longitudinal fasciculus) and in the left temporal-parietal white matter pathway (inferior longitudinal fasciculus) compared to controls, which were correlated with speed of reading, spel- 
ling, and pseudoword decoding (Deutsch et al., 2005; Klingberg et al., 2000; Niogi \& Mc Candliss, 2006; Rimrodt, Peterson, Denckla, Kaufmann, \& Cutting, 2009; Steinbrink et al., 2008; Thomason \& Thompson, 2011). Concurrent to these intra-hemispheric fiber pathway differences, DTI studies suggest that the fiber orientation in the right superior longitudinal fasciculus differs in individuals with dyslexia, with an increased number in the superior-inferior orientation in its temporal-parietal projection as compared to controls, whose fibers are oriented anterior-laterally (Carter et al., 2009). Such differences in fiber orientation in the superior longitudinal fasciculus, for example, may account for the differences in fiber connectivity between the frontal and temporal cortices.

The intra-hemispheric differences in coherence activity and functional connectivity in the right and left frontal lobe are likely to be mediated short frontal lobe connections in the fronto-orbitopolar tract that connect the posterior orbitofrontal cortex with the anterior polar region and the frontal superior longitudinal fasciculus (Catani et al., 2012). In addition, there is a more complex system of U-shaped fibers in the regions of the central, precentral, perinsular, and fronto-marginal sulcus (Catani et al., 2012).

A qualitative and quantitative review by Vandermosten and colleagues (2012) of the diffusion tensor imaging literature in dyslexia suggest that lower FA values in the left temporoparietal and frontal areas are indicative of poorer reading ability and that most of these regions coincide with the left arcuate fasciculus (superior longitudinal fasciculus) and corona radiata, with comparatively few studies showing a role for the posterior part of the corpus callosum or more ventral tracts as the inferior longitudinal fasciculus and the inferior fronto-occipital fasciculus. The conclusions of Vandermosten and colleagues (2012) are entirely consistent with those of the present study and provide a neuroanatomical framework for our intra-hemispheric MEG coherence and functional connectivity results.

\subsection{Role of the Right Orbitofrontal Cortex and Other Frontal Cortical Areas in Dyslexia}

Of the 69 synchronous oscillatory active brain region pairs that differentiated individuals with dyslexia from controls in the SWM task, the majority were frontocortical areas, with a substantial number of these be orbitofrontal regions. The results suggested an overall reduced coherence in the right frontal cortex in individuals with dyslexia performing a spatial working memory task, with a convergence of evidence suggesting a lower connectivity specifically in the right middle orbitofrontal gyrus and the right lateral orbitofrontal gyrus and their intra-hemispheric local pathways (fronto-orbitopolar, fronto-marginal, frontal longitudinal, and uncinate), long pathways (superior longitudinal fasciculus, inferior fronto-occipital fasciculus), and inter-hemispheric frontal and temporal lobe connections (e.g. anterior and midbody callosal radiations).

Functionally distinct pathways for auditory processing in the orbitofrontal cortex include a rostral stream associated with phonetic processing and a more caudal stream terminating just posterior to the orbitofrontal cortex in the periarcuate prefrontal cortex associated with auditory-spatial processing. Both ventral and dorsal visual streams share connections with orbitofrontal cortical areas, including rich projections to and from the superior temporal gyrus, important for integration of spatial and object processing (Cavada, Company, Tejedor, CruzRizzolo, \& Reinoso-Suarez, 2000) and the only region in the present study to demonstrate a reduced amplitude of activation in individuals with dyslexia during both verbal and spatial working memory paradigms. Meta-analysis of functional imaging studies and selective lesions suggests that it's the medial orbitofrontal cortex that is related to the initial evaluation of the affective or motivational significance of stimuli, monitoring, learning, and memory of the reward value of reinforcers, whereas the lateral orbitofrontal is related to the evaluation of punishers, the reappraisal of emotional significance of stimuli, and response suppression, which may lead to a change in ongoing behavior (Stuss \& Levine, 2002; Happaney, Zelazo, \& Stuss, 2004; Kringelbach \& Rolls, 2004).

Of particular clinical importance is the potential use of evoked MEG coherence and connectivity in the right medial and lateral orbitofrontal gyri as a diagnostic marker for dyslexia. Logistic regression of the coherence values by group membership was significant, with an overall predictive success of $84.4 \%$ (88.9\% for controls and $\mathbf{7 7 . 8 \%}$ for dyslexics). Coherence activity or functional connectivity in the right lateral orbitofrontal gyrus and right middle orbitofrontal gyrus region pair substantially contributed to group membership such that the forward addition of other pathways failed to significantly add to the predictive value of the model. Further, there was a significant positive linear correlation between the coherence of the right lateral and right middle orbitofrontal gyri and phonological decoding when all three frequency ranges were assessed and when just the gamma 
coherence frequency in this functional pathway was correlated with phonological decoding abilities.

\subsection{Diagnostic Implications}

\subsubsection{Comorbidity}

Dysregulation and reduced functional connectivity in the right medial and right lateral orbitofrontal gyri and their intra-hemispheric, inter-hemispheric, and subcortical connections likely contribute to the high comorbidity of dyslexia with other psychiatric disorders such as Attention Deficit Hyperactivity Disorder (ADHD), anxiety disorders, and mood disorders (Carroll, Maughan, Goodman, \& Meltzer, 2005; German, Gagliano, \& Curatolo, 2010; Hinshaw, 1992; Taurines et al., 2010; Trzesniewski, Moffitt, Caspi, Taylor, \& Maughan, 2006; Willcutt et al., 2010b) that have been hypothesized to involve the dysregulation of neural reward circuits mediating motivation and impulsivity that include the orbitofrontal cortex (Katz, Brown, Roth, \& Beers, 2011; Kerestes et al., 2012; Toplak, Jain, \& Tannock, 2005). In addition to such shared neuroanatomical dysregulation, multiple common genetic loci (Willcutt et al., 2010a, 2010b) and gene $\times$ environment $(G \times E)$ interactions (Pennington et al., 2009), particularly around susceptibility chromosomes $6 \mathrm{p}, 15 \mathrm{q}$, and 18p, are common to both dyslexia and ADHD.

\subsubsection{Importance of Early Diagnosis}

Randomized control studies have consistently shown that reading instruction needs to be intensive (e.g., 120 minutes per day for 8 weeks), occur in small groups of 1 or 2 students per teacher, and include explicit and systematic instruction in phonological awareness and decoding strategies to be effective in improving reading accuracy and fluency (Alexander \& Slinger-Constant, 2004; Keller \& Just, 2009; Snowling \& Hulme, 2011; Strong, Torgerson, Torgerson, \& Hulme, 2011). Even with such intensive, evidence-based instruction, there are children who fail to benefit from reading remediation. Further, the gains typically achieved from such reading programs are maintained for one to two years in approximately half of the children who return to their school's standard curriculum. Such improvements are much more likely to occur in children who are beginning to read (ages 6 to 8) than in older children and are much more difficult to achieve for reading fluency than for accuracy. Thus, these resource-demanding interventions are effective for many children, but there are still challenges in developing early diagnostic methods, strategies for prevention, and treatments that are effective in a broader range of children and adolescents.

Applications of the current evoked MEG coherence findings in the right orbitofrontal cortex in dyslexia could serve as the basis for an early diagnostic strategy that could be implemented prior to the development of reading. As a prospectively defined demonstration study, children with a familial risk for developing dyslexia would be screened using MEG imaging during the performance of an age-appropriate spatial working memory task. Instead of employing an n-2 back task used in the present study, an n-1 back spatial task could be substituted in the behavioral-imaging screen. In conjunction with MEG imaging, those children that demonstrating a reduced connectivity in the right orbitofrontal cortex would be predicted to be at a heightened risk of developing dyslexia.

\subsubsection{Prevention}

Hoeft and colleagues $(2007,2011)$ examined the utility of behavioral (standardized tests) and functional and structural neuroimaging measures taken with children ( $8-12$ years of age) at the beginning of a school year for predicting their decoding ability at the end of that school year. Specific patterns of brain activation during phonological processing and morphology, revealed by voxel-based morphometry (VBM) of gray and white matter densities, predicted later decoding ability. Standardized behavioral measures of reading and language yielded a behavioral model that accounted for $65 \%$ of the variance in end of the year performance on a measure of phonological decoding (Woodcock-Johnson Word Attack subtest). Brain imaging measures consisting of both fMRI and DTI yielded a neuroimaging model that accounted for $57 \%$ of the end-of-the-year variance in phonological decoding. However, it was the combined model of behavioral and neuroimaging measures that was the most predictive of decoding skills, explaining $81 \%$ of the variance. These findings suggest that neuroimaging methods may be useful in enhancing the early identification of children at risk for poor decoding and reading skills.

Similarly, a spatial working memory task in conjunction with MEG imaging of the right orbitofrontal cortex could be used not only to predict children who were at risk of developing dyslexia but also to signal the initiation of an intervention to prevent the development of the disorder, targeting children who are vulnerable at the 
time when treatment is likely to be the most efficacious. Such a strategy holds the potential of reducing the alarming rates of dyslexia and the years of emotional and psychological distress, that is associated with the disorder.

\subsubsection{Implications for Treatment and Intervention}

Traditional reading remediation or intervention programs for individuals with dyslexia have exclusively focused on phonological awareness and decoding strategies (Alexander \& Slinger-Constant, 2004; Keller \& Just, 2009; Snowling \& Hulme, 2011; Strong et al., 2011). Rarely do these programs address the subprocesses and functions that underlie these behaviors and, more broadly, reading. The present coherence activity study in dyslexia, as well as numerous others (i.e. Eden et al., 2004; Eden et al., 1996; Galaburda \& Livingstone, 1993), have clearly implicated the fundamental importance of spatial working memory in reading. Individuals with dyslexia refractory to remediation or intervention may have fundamental deficits in complex attention and visual working memory, which may not be directly addressed in reading programs. Bacon, Parmentier, \& Barr (2012), in fact, recently demonstrated that a consistent visual spatial deficit in adult dyslexics, in performing a Corse block task backwards, could be ameliorated by visual strategy instruction.

\section{Limitations}

A number of limitations need to be considered in the interpretation of these results. The study included a comparatively small number of subjects: a total of nine neurotypical controls and seven individuals with dyslexia. While the results were sufficiently robust to demonstrate significant statistical differences between individuals with dyslexia and controls, the generalizability of these findings may be limited, as they may not apply to a larger population. The subjects were largely male, and the mean ages of the dyslexia and control groups were 24 and 26 years of age, respectively. While the participants in the study were matched for age, gender, and IQ, the results may be particularly applicable to an adolescent-adult male population. The subjects in this study had an above-average IQ (Dyslexics = 112 FSIQ, Controls = 115 FSIQ), which may reflect a bias in the selection process.

To be included in the study, individuals suspected of dyslexia underwent a neuropsychological evaluation were diagnosed with a reading disorder if their performance on a standardized reading measure was in the lower $25^{\text {th }}$ percentile or their performance on a standardized reading measure was at least one standard deviation below their standard IQ score. Both of these entry criteria indicate clinical difficulties with reading and are consistent with the DSM-5 (American Psychiatric Association, 2013) diagnostic criteria of a reading learning disorder but may have added to the variability of the results. The present study was insufficiently powered to identify differences that may exist between such differentially defined subsets of individuals with dyslexia and to determine whether the results reflect primary reading deficiencies or a combination of such deficiencies and levels of compensation.

Neuroimaging studies comparing a clinical population to controls are inherently limited, as they are correlational. The present MEG coherence study is no exception and should not be misinterpreted as implying a causative link between brain activation and connectivity and the etiology of dyslexia. To address this and the other limitations delineated above, future studies will need to be prospectively defined and use a double-dissociative design, such as assigning individuals to the dyslexia or control groups a priori based on their coherence activity in the right medial and lateral orbitofrontal gyri during a spatial working memory task. If the functional connectivity in the right orbitofrontal cortex is a determinant of dyslexia, those with reduced coherence activity within this region would be predicted to have a reading disorder on standardized measures, while those individuals whose coherence activity within the right orbitofrontal gyri was within normal limits when performing this spatial working memory task would be unlikely to have a reading disorder. The study will also need to be sufficiently powered to dissociate contributing factors (e.g., age, gender, IQ, current reading abilities, etc.) to facilitate the generalizability of the results.

Finally, the present study focused on the lower end $(30-45 \mathrm{~Hz})$ of the gamma frequency range that extends from $30-100 \mathrm{~Hz}$. While the findings may vary at the higher end of the gamma frequency band (above $45 \mathrm{~Hz}$ ), gamma activation is generally most robust at $40 \mathrm{~Hz}$ during the performance of high-order language tasks (Weiss \& Mueller, 2003), with frequencies of $60 \mathrm{~Hz}$ and higher often introducing recording artifacts. 


\section{Conclusion}

The results of these studies are consistent with and extend our understanding of the pathophysiological and psychobiological bases of dyslexia. MEG neuroimaging during the performance of orthographic and non-orthographic working memory tasks suggests fronto-temporal inefficiencies/impairments in individuals with dyslexia as evidenced by the early onset and reliance on prefrontal cortical areas, the differential activation of fronto-temporal brain systems, and the pattern of functional connectivity of the fronto-temporal pathways mediating these behaviors. MEG coherence analysis in individuals with dyslexia suggested a dysregulation and a lower connectivity in functional circuits of the 1) right frontal, 2) right fronto-temporal, 3) left and right frontal, 4) left temporal and right frontal, and 5) left occipital and right frontal, consistent with deficits in both intra- and inter-hemispheric integration and communication. These functional connectivity findings in dyslexia complement the neuroanatomical findings that report a reduced DTI coherence in the intra-hemispheric (fronto-orbitopolar, superior longitudinal fasciculus, and inferior fronto-occipital fasciculus) and inter-hemispheric (corona radiata, corpus callosal fibers in the genu and mid-body/isthmus regions) tracts. The present studies highlight the importance of working memory and the functional connectivity of right orbitofrontal cortex and its frontal and temporal lobe projections in dyslexia, with ramifications for prevention, early diagnosis, and the development of effective, evidence-based treatments and interventions.

\section{References}

Alexander, A., \& Slinger Constant, A. (2004). Current Status of Treatments for Dyslexia: Critical Review. Journal of Child Neurology, 19, 744-758.

American Psychiatric Association (2013). Diagnostic and Statistical Manual of Mental Disorders (5th ed.). Arlington, VA: American Psychiatric Publishing.

Arns, M., Peters, S., Breteler, R., \& Verhoeven, L. (2007). Different Brain Activation Patterns in Dyslexic Children: Evidence from EEG Power and Coherence Patterns for the Double-Deficit Theory of Dyslexia. Journal of Integrative Neuroscience, 6, 175-190. http://dx.doi.org/10.1142/S0219635207001404

Bacon, A. M., Parmentier, F. B. R., \& Barr, P. (2012). Visuospatial Memory in Dyslexia: Evidence for Strategic Deficits. Memory, 20, 1-21.

Baddeley, A. D. (2007). Working Memory, Thought \& Action. Oxford, UK: Oxford University Press. http://dx.doi.org/10.1093/acprof:oso/9780198528012.001.0001

Bailey, P., \& Snowling, M. (2002). Auditory Processing and the Development of Language and Literacy. British Medical Bulletin, 63, 135-146. http://dx.doi.org/10.1093/bmb/63.1.135

Belardinelli, P., Ciancetta, L., Staud, M., Pizzella, V., Londei, A., Birbaumer, N. et al. (2007). Cerebro-Muscular and Cerebro-Cerebral Coherence in Patients with Pre- and Perinatally Acquired Unilateral Brain Lesions. NeuroImage, 37, 13011314. http://dx.doi.org/10.1016/j.neuroimage.2007.05.053

Benjamini, Y., \& Hochberg, Y. (1995). Controlling the False Discovery Rate: A Practical and Powerful Approach to Multiple Testing. Journal of the Royal Statistical Society, 57, 289-300.

Benton, A. L. (1975). Developmental Dyslexia: Neurological Aspects. Advances in Neurology, 7, 1-47.

Berninger, V., Raskind, W., Richards, T., Abbott, R., \& Stock, P. (2008). A Multidisciplinary Approach to Understanding Developmental Dyslexia within Working-Memory Architecture: Genotypes, Phenotypes, Brain and Instruction. Developmental Neuropsychology, 33, 707-744. http://dx.doi.org/10.1080/87565640802418662

Bishop, D. V. M., \& Snowling, M. (2004). Developmental Dyslexia and Specific Language Impairment: Same or Different? Psychological Bulletin, 130, 858-886. http://dx.doi.org/10.1037/0033-2909.130.6.858

Boisgueheneuc, F., Levy, R., Volle, E., Seassau, M., Duffau, H., Kinkingnehun, S. et al. (2006). Functions of the Left Superior Frontal Gyrus in Humans: A Lesion Study. Brain, 129, 3315-3328. http://dx.doi.org/10.1093/brain/awl244

Bowyer, S. M., Mason, K., Tepley, N., Smith, B., \& Barkley, G. L. (2003). Magnetoencephalographic Validation Parameters for Clinical Evaluation of Interictal Epileptic Activity. Neurophysiology, 20, 87-93. http://dx.doi.org/10.1097/00004691-200304000-00001

Bowyer, S. M., Moran, J. E., Mason, K. M., Constantinou, J. E., Smith, B. J., Barkley, G. L. et al. (2004). MEG Localization of Language-Specific Cortex Utilizing MR-FOCUSS. Neurology, 62, 2247-2255.

http://dx.doi.org/10.1212/01.WNL.0000130385.21160.7A

Carroll, J. M., Maughan, B., Goodman, R., \& Meltzer, H. (2005). Literacy Difficulties and Psychiatric Disorders: Evidence for Comorbidity. Developmental Psychology, 46, 524-532. 
Carter, J., Lanham, D., Cutting, L., Clements Stephens, A., Chen, X., Hadzipasic, M. et al. (2009). A Dual DTI Approach to Analyzing White Matter in Children with Dyslexia. Psychiatry Research, 172, 215-219. http://dx.doi.org/10.1016/j.pscychresns.2008.09.005

Catani, M., Dell'Acqua, F., Vergani, F., Malik, F., Hodge, H., Roy, P. et al. (2012). Short Frontal Lobe Connections of the Human Brain. Cortex, 48, 273-291. http://dx.doi.org/10.1016/j.cortex.2011.12.001

Cavada, C., Company, T., Tejedor, J., Cruz-Rizzolo, R. J., \& Reinoso-Suarez, F. (2000). The Anatomical Connections of the Macaque Monkey Orbitofrontal Cortex: A Review. Cerebral Cortex, 10, 220-242. http://dx.doi.org/10.1093/cercor/10.3.220

Conway, T., Heilman, K., Gopinath, K., Peck, K., Bauer, R., Briggs, R. et al. (2008). Neural Substrates Related to Auditory Working Memory Comparisons in Dyslexia: An fMRI Study. Journal of the International Neuropsychological Society, 14, 629-639. http://dx.doi.org/10.1017/S1355617708080867

Corina, D. P., Richards, T. L., Serafini, S., Richards, A. L., Steury, K., Abbott, R. D. et al. (2001). fMRI Auditory Language Differences between Dyslexic and Able Reading Children. Neuroreport, 12, 1195-1201. http://dx.doi.org/10.1097/00001756-200105080-00029

Cropley, V. L., Fujita, M., Innis, R. B., \& Nathan, P. J. (2006). Molecular Imaging of the Dopaminergic System and Its Association with Human Cognitive Function. Biological Psychiatry, 59, 898-907. http://dx.doi.org/10.1016/j.biopsych.2006.03.004

Crosson, B. (1999). Subcortical Mechanisms in Language: Lexical-Semantic Mechanisms and the Thalamus. Brain and Cognition, 40, 414-438. http://dx.doi.org/10.1006/brcg.1999.1088

Crosson, B., Benefield, H., Cato, M. A., Sadek, J. R., Moore, A. B., \& Wierenga, C. E. et al. (2003). Left and Right Basal Ganglia and Frontal Activity during Language Generation: Contributions to Lexical, Semantic and Phonological Processes. Journal of the International Neuropsychological Society, 9, 1061-1077. http://dx.doi.org/10.1017/S135561770397010X

D'Esposito, M., Aguirre, G. K., Zarahn, E., Ballard, D., Shin, R. K., \& Lease, J. (1998). Functional MRI Studies of Spatial and Nonspatial Working Memory. Cognitive Brain Research, 7, 1-13. http://dx.doi.org/10.1016/S0926-6410(98)00004-4

Deutsch, G. K., Dougherty, R. F., Bammer, R., Siok, W. T., Gabrieli, J. D., \& Wandell, B. (2005). Children’s Reading Performance Is Correlated with White Matter Structure Measured by Diffusion Tensor Imaging. Cortex, 41, 354-363. http://dx.doi.org/10.1016/S0010-9452(08)70272-7

Dong, Y., Nakamura, K., Okada, T., Hanakawa, T., Fukuyama, H., Mazziotta, J. C. et al. (2005). Neural Mechanisms Underlying the Processing of Chinese Words: An fMRI Study. Neuroscience Research, 52, 139-145. http://dx.doi.org/10.1016/j.neures.2005.02.005

Eden, G. F., Jones, K. M., Cappell, K., Gareau, L., Wood, F. B., Zeffiro, T. A. et al. (2004). Neural Changes Following Remediation in Adult Developmental Dyslexia. Neuron, 44, 411-422. http://dx.doi.org/10.1016/j.neuron.2004.10.019

Eden, G. F., Van Meter, J. W., Rumsey, J. M., \& Zeffiro, T. A. (1996). The Visual Deficit Theory of Developmental Dyslexia. NeuroImage, 4, S108-S117. http://dx.doi.org/10.1006/nimg.1996.0061

Efron, B. (2010). Large-Scale Inference: Empirical Bayes Methods for Estimation, Testing and Prediction. Cambridge: Cambridge University Press. http://dx.doi.org/10.1017/CBO9780511761362

Elisevich, K., Shukla, N., Moran, J., Smith, B., Schultz, L., Mason, K. et al. (2011). An Assessment of MEG Coherence Imaging in the Study of Temporal Lobe Epilepsy. Epilepsia, 52, 1110-1119. http://dx.doi.org/10.1111/j.1528-1167.2011.02990.x

Facoetti, A., Trussardi, A. N., Ruffino, M., Lorusso, M. L., Cattaneo, C., Galli, R. et al. (2010). Multisensory Spatial Attention Deficits Are Predictive in Phonological Decoding Skills in Developmental Dyslexia. Journal of Cognitive Neuroscience, 22, 1011-1025. http://dx.doi.org/10.1162/jocn.2009.21232

Farris, E. A., Odegard, T. N., Miller, H. L., Ring, J., Allen, G., \& Black, J. (2011). Functional Connectivity between the Left and Right Inferior Frontal Lobes in a Small Sample of Children with and without Reading Difficulties. Neurocase, 17, 425-439. http://dx.doi.org/10.1080/13554794.2010.532141

Fiez, J., Tranel, D., Seager Frerichs, D., \& Damasio, H. (2006). Specific Reading and Phonological Processing Deficits Are Associated with Damage to the Left Frontal Operculum. Cortex, 42, 624-643. http://dx.doi.org/10.1016/S0010-9452(08)70399-X

Fine, J. G., Semrud-Clikeman, M., Keith, T. Z., Stapleton, L. M., \& Hynd, G. W. (2007). Reading and the Corpus Callosum: An MRI Family Study of Volume and Area. Neuropsychology, 21, 235-241. http://dx.doi.org/10.1037/0894-4105.21.2.235

Fletcher, J. M., Lyon, G. R., Fuchs, L., \& Barnes, M. (2007). Learning Disabilities: From Identification to Intervention. New York: Guilford Press.

Gaillard, W. D., Balsamo, L. M., Ibrahim, Z., Sachs, B. C., \& Xu, B. (2003). fMRI Identifies Regional Specialization of Neural Networks for Reading in Young Children. Neurology, 60, 94-100. http://dx.doi.org/10.1212/WNL.60.1.94 
Galaburda, A., \& Livingstone, M. (1993). Evidence for a Magnocellular Defect in Developmental Dyslexia. Annals of the New York Academy of Sciences, 682, 70-82. http://dx.doi.org/10.1111/j.1749-6632.1993.tb22960.x

German, E., Gagliano, A., \& Curatolo, P. (2010). Comorbidity of ADHD and Dyslexia. Developmental Neuropsychology, 35, 475-493. http://dx.doi.org/10.1080/87565641.2010.494748

Gevins, A. S., \& Cutillo, B. S. (1993). Neuroelectric Evidence for Distributed Processing in Human Working Memory. Electroencephalography and Clinical Neurophysiology, 87, 128-143. http://dx.doi.org/10.1016/0013-4694(93)90119-G

Happaney, K., Zelazo, P. D., \& Stuss, D. T. (2004). Development of Orbitofrontal Function: Current Themes and Future Directions. Brain and Cognition, 55, 1-10. http://dx.doi.org/10.1016/j.bandc.2004.01.001

Hasan, K. M., Molfese, D. L., Wallmuni, I. S., Stuebing, K. K., Papanicolaou, A. C., Narayana, P. A., \& Fletcher, J. M. (2012). Diffusion Tensor Quantification and Cognitive Correlates of the Macrostructure and Microstructure of the Corpus Callosum in Typically Developing and Dyslexic Children. NMR Biomedicine, 25, 1263-1270.

Helenius, P., Tarkiainen, A., Cornelissen, P., Hansen, P. C., \& Salmelin, R. (1999). Dissociation of Normal Feature Analysis and Deficient Processing of Letter-Strings in Dyslexic Adults. Cerebral Cortex, 9, 476-483.

http://dx.doi.org/10.1093/cercor/9.5.476

Hinshaw, S. P. (1992). Externalizing Behavior Problems and Academic Underachievement in Childhood and Adolescence: Causal Relationships and Underlying Mechanisms. Psychological Bulletin, 111, 127-155. http://dx.doi.org/10.1037/0033-2909.111.1.127

Hoeft, F., Mc Candliss, B. D., Black, J. M., Gantman, A., Zakerani, N., Hulme, C. et al. (2011). Neural Systems Predicting Long-Term Outcome in Dyslexia. Proceedings of the Academy of Science of the United States of America, 108, 361-368.

Hoeft, F., Meyler, A., Hernandez, A., Juel, C., Taylor Hill, H., Martindale, J. et al. (2007). Functional and Morphometric Brain Dissociation between Dyslexia and Reading Ability. Proceedings of the National Academy of Sciences of the United States of America, 104, 4234-4239. http://dx.doi.org/10.1073/pnas.0609399104

Hoeft, F., Ueno, T., Reiss, A. L., Meyler, A., Whitfield-Gabrieli, S., Glover, G. H. et al. (2007). Prediction of Childrens Reading Skills Using Behavioral, Functional and Structural Neuroimaging Measures. Behavioral Neuroscience, 12, 602613. http://dx.doi.org/10.1037/0735-7044.121.3.602

Hynd, G. W., Hall, J., Novey, E. S., Eliopulos, D., Black, K., Gonzalez, J. J. et al. (1995). Dyslexia and Corpus Callosum Morphology. Archives of Neurology, 52, 32-38. http://dx.doi.org/10.1001/archneur.1995.00540250036010

Indefrey, P., \& Levelt, W. J. (2004). The Spatial and Temporal Signatures of Word Production Components. Cognition, 92, 101-144. http://dx.doi.org/10.1016/j.cognition.2002.06.001

Karnath, H. O. (2001). New Insights into the Functional of the Superior Temporal Cortex. Nature Reviews Neuroscience, 2, 568-576. http://dx.doi.org/10.1038/35086057

Katz, L. J., Brown, F. C., Roth, R. M., \& Beers, S. R. (2011). Processing Speed and Working Memory Performance in Those with Both ADHD and a Reading Disorder Compared with Those with ADHD Alone. Archives of Clinical Neuropsychology, 26, 425-433. http://dx.doi.org/10.1093/arclin/acr026

Keller, T., \& Just, M. (2009). Altering Cortical Connectivity: Remediation-Induced Changes in the White Matter of Poor Readers. Neuron, 64, 624-631. http://dx.doi.org/10.1016/j.neuron.2009.10.018

Kerestes, R., Ladouceur, C. D., Meda, S., Nathan, P. J., Blumberg, H. P., Maloney, K. et al. (2012). Abnormal Prefrontal Activity Subserving Attentional Control of Emotion in Remitted Depressed Patients during a Working Memory Task with Emotional Distractors. Psychological Medicine, 42, 29-40. http://dx.doi.org/10.1017/S0033291711001097

Kibby, M., Kroese, J., Krebbs, H., Hill, C., \& Hynd, G. (2009). The Pars Triangularis in Dyslexia and ADHD: A Comprehensive Approach. Brain and Language, 111, 46-54. http://dx.doi.org/10.1016/j.bandl.2009.03.001

Klingberg, T., Hedehus, M., Temple, E., Salz, T., Gabrieli, J. D., Moseley, M. E. et al. (2000). Microstructure of Temporo-Parietal White Matter as a Basis for Reading Ability: Evidence from Diffusion Tensor Magnetic Resonance Imaging. Neuron, 25, 483-500. http://dx.doi.org/10.1016/S0896-6273(00)80911-3

Kringelbach, M. L., \& Rolls, E. T. (2004). The Functional Neuroanatomy of the Human Orbitofrontal Cortex: Evidence from Neuroimaging and Neuropsychology. Progress in Neurobiology, 72, 341-372. http://dx.doi.org/10.1016/j.pneurobio.2004.03.006

Kujala, J., Pammer, K., Cornelissen, P. L., Roebroeck, P., Formisano, E., \& Salmelin, R. (2007) Phase Coupling in a Cerebro-Cerebellar Network at 8-13 Hz during Reading. Cerebral Cortex, 17, 1476-1485. http://dx.doi.org/10.1093/cercor/bhl059

Laine, M., Salmelin, R., Helenius, P., \& Marttila, R. (2000). Brain Activation during Reading in Deep Dyslexia: An MEG Study. Journal of Cognitive Neuroscience, 12, 622-634. http://dx.doi.org/10.1162/089892900562381

Lajiness-O’Neill, R. Akamine, Y., \& Bowyer, S. M. (2007). Treatment Effects of Fast for Word Demonstrated by Magnetoencephalography in a Child with Developmental Dyslexia. Neurocase, 13, 390-401. 
Machizawa, M., Kanai, R., Rees, G., \& Driver, J. (2010). Cortical Anatomy Relates to Individual Differences in Dissociable Aspects of Attention and Visual Working Memory Capacity. Journal of Vision, 10, 775. http://dx.doi.org/10.1167/10.7.775

Mc Candliss, B., \& Noble, K. (2003). The Development of Reading Impairment: A Cognitive Neuroscience Model. Mental Retardation and Developmental Disabilities Research Reviews, 9, 196-204. http://dx.doi.org/10.1002/mrdd.10080

Moran, J. E. (2008). MEG Tools. Detroit, MI: OU-HFH. http://www.megimaging.com

Moran, J. E., Bowyer, S. M., \& Tepley, N. (2005). Multi-Resolution FOCUSS: A Source Imaging Technique Applied to MEG Data. Brain Topography, 18, 1-17. http://dx.doi.org/10.1007/s10548-005-7896-X

Nagarajan, S., Mahncke, H., Salz, T., Tallal, P., Roberts, T., \& Merzenich, M. M. (1999). Cortical Auditory Signal Processing in Poor Readers. Proceedings of the National Academy of Sciences of the United States of America, 96, 64836488. http://dx.doi.org/10.1073/pnas.96.11.6483

Newbury, D., Bishop, D. V. M., \& Monaco, A. (2005). Genetic Influences on Language Impairment and Phonological Short-Term Memory. Trends in Cognitive Sciences, 9, 528-534. http://dx.doi.org/10.1016/j.tics.2005.09.002

Nicolson, R. I., Fawcett, A. J., Brookes, R. L., \& Needle, J. (2010). Procedural Learning and Dyslexia. Dyslexia, 16, 194212. http://dx.doi.org/10.1002/dys.408

Niogi, S. N., \& Mc Candliss, B. D. (2006). Left Lateralized White Matter Microstructure Accounts for Individual Differences in Reading Ability and Disability. Neuropsychologia, 44, 2178-2188. http://dx.doi.org/10.1016/j.neuropsychologia.2006.01.011

Pammer, K., Hansen, P., Holliday, I., \& Cornelissen, P. (2006). Attentional Shifting and the Role of the Dorsal Pathway in Visual Word Recognition. Neuropsychologia, 44, 2926-2936. http://dx.doi.org/10.1016/j.neuropsychologia.2006.06.028

Papanicolaou, A. C., Simos, P. G., Breier, J. I., Fletcher, J. M., Foorman, B. R., Francis, D. et al. (2003). Brain Mechanisms for Reading in Children with and without Dyslexia: A Review of Studies of Normal Development and Plasticity. Developmental Neuropsychology, 24, 593-612. http://dx.doi.org/10.1080/87565641.2003.9651912

Pennington, B., McGrath, L., Rosenberg, J., Barnard, H., Smith, S., Willcutt, E. et al. (2009). Gene X Environment Interactions in Reading Disability and Attention-Deficit/Hyperactivity Disorder. Developmental Psychology, 45, 77-89. http://dx.doi.org/10.1037/a0014549

Richlan, F. (2012). Developmental Dyslexia: Dysfunction of the Left Hemisphere-Reading Network. Frontier in Human Neuroscience, 6, 1-5.

Richlan, F., Kronbichler, M., \& Wimmer, H. (2009). Functional Abnormalities in the Dyslexic Brain: A Quantitative Meta-Analysis of Neuroimaging Studies. Human Brain Mapping, 30, 3299-3308. http://dx.doi.org/10.1002/hbm.20752

Rimrodt, S. L., Peterson, D. J., Denckla, M. B., Kaufmann, W. E., \& Cutting, L. E. (2009). White Matter Microstructural Differences Linked to Left Perisylvian Language Network in Children with Dyslexia. Cortex, 46, 739-749. http://dx.doi.org/10.1016/j.cortex.2009.07.008

Robichon, F., Bouchard, P., Demonet, J., \& Habib, M. (2000). Developmental Dyslexia: Re-Evaluation of the Corpus Callosum in Male Adults. European Neurology, 43, 233-237. http://dx.doi.org/10.1159/000008182

Sarkari, S., Simos, P., Fletcher, J., Castillo, E., Breier, J., \& Papanicolaou, A. (2002). Contributions of Magnetic Source Imaging to the Understanding of Dyslexia. Seminars in Pediatric Neurology, 9, 229-238.

http://dx.doi.org/10.1053/spen.2002.35506

Sekihara, K., Nagarajan, S. S., Poeppel, D., Marantz, A., \& Miyashita, Y. (2001). Reconstructing Saptio-Temporal Activities of Neural Sources Using MEG Vector Beamformer Technique. IEEE Transactions on Biomedical Engineering, 48, 760771. http://dx.doi.org/10.1109/10.930901

Shaywitz, B. A., Shaywitz, S. E., Blachman, B. A., Pugh, K. R., Fulbright, R. K., Skudlarski, P. et al. (2004). Development of Left Occipito-Temporal Systems for Skilled Reading in Children after a Phonologically-Based Intervention. Biological Psychiatry, 55, 926-933. http://dx.doi.org/10.1016/j.biopsych.2003.12.019

Shaywitz, S. E., Shaywitz, B. A., Pugh, K. R., Fulbright, R. K., Constable, R. T., Mencl, W. E. et al. (1998). Functional Disruption in the Organization of the Brain for Reading in Dyslexia. Proceedings of the National Academy of Sciences of the United States of America, 95, 2636-2641. http://dx.doi.org/10.1073/pnas.95.5.2636

Simos, P. G., Breier, J. I., Zouridakis, G., \& Papanicolaou, A. C. (1998). Identification of Language-Specific Brain Activity Using Magnetoencephalography. Journal of Clinical and Experimental Neuropsychology, 20, 706-722.

http://dx.doi.org/10.1076/jcen.20.5.706.1127

Simos, P. G., Papanicolaou, A. C., Breier, J. I., Fletcher, J. M., Wheless, J. W., Maggio, W. W. et al. (2000). Insights into Brain Function and Neural Plasticity Using Magnetic Source Imaging. Journal of Clinical Neurophysiology, 17, $143-162$. http://dx.doi.org/10.1097/00004691-200003000-00004

Simos, P., Fletcher, J., Denton, C., Sarkari, S., Billingsley Marshall, R., \& Papanicolaou, A. (2006). Magnetic Source Imag- 
ing Studies of Dyslexia Interventions. Developmental Neuropsychology, 30, 591-611.

http://dx.doi.org/10.1207/s15326942dn3001_4

Simos, P., Fletcher, J., Foorman, B. R., Francis, D. J., Castillo, E. M., Davis, R. N. et al. (2002). Brain Activation Profiles during Early Stages of Reading Acquisition. Journal of Child Neurology, 17, 159-163.

http://dx.doi.org/10.1177/088307380201700301

Snowling, M., \& Hulme, C. (2011). Evidence-Based Interventions for Reading and Language Difficulties: Creating a Virtuous Circle. British Journal of Educational Psychology, 81, 1-23. http://dx.doi.org/10.1111/j.2044-8279.2010.02014.x

Sridharan, D., Levitin, D. J., \& Menon, V. (2008). A Critical Role for the Fronto-Insular Cortex in Switching between Central-Executive and Default-Mode Networks. Proceedings of the National Academy of Sciences of the United States of America, 105, 12569-12574. http://dx.doi.org/10.1073/pnas.0800005105

Steinbrink, C., Vogt, K., Kastrup, A., Müller, H. P., Juengling, F. D., Kassubek, J. et al. (2008). The Contribution of White and Gray Matter Differences to Developmental Dyslexia: Insights from DTI and VBM at 3.0 T. Neuropsychologica, 46, 3170-3178. http://dx.doi.org/10.1016/j.neuropsychologia.2008.07.015

Strong, G., Torgerson, C., Torgerson, D., \& Hulme, C. (2011). A Systematic Meta-Analytic Review of Evidence for the Effectiveness of the "Fast for Word” Language Intervention Program. Journal of Child Psychology and Psychiatry and Allied Disciplines, 52, 224-235. http://dx.doi.org/10.1111/j.1469-7610.2010.02329.x

Stuss, D. T. (2011). Function of the Frontal Lobes: Relation to Executive Functions. Journal of the International Neuropsychological Society, 17, 759-765. http://dx.doi.org/10.1017/S1355617711000695

Stuss, D. T., \& Alexander, M. P. (2007). Is There a Dysexecutive Syndrome? Philosophical Transactions of the Royal Society, 362, 901-915. http://dx.doi.org/10.1098/rstb.2007.2096

Stuss, D. T., \& Levine, B. (2002). Adult Clinical Neuropsychology: Lessons from Studies of the Frontal Lobes. Annual Reviews in Psychology, 53, 401-433. http://dx.doi.org/10.1146/annurev.psych.53.100901.135220

Swanson, H. L., Howard, C. B., \& Saez, L. (2006). Do Different Components of Working Memory Underlie Different Subgroups of Reading Disabilities? Journal of Learning Disabilities, 39, 252-269. http://dx.doi.org/10.1177/00222194060390030501

Talcott, J. B., Witton, C., McLean, M. F., Hansen, P. C., Rees, A., Green, G. G. et al. (2000). Dynamic Sensory Sensitivity of Children's Word Decoding Skills. Proceeding of the National Academy of Sciences of the United States of America, 97, 2952-2957.

Tarkiainen, A., Helenius, P., \& Salmelin, R. (2003). Category-Specific Occipitotemporal Activation during Face Perception in Dyslexic Individuals: An MEG Study. Neuroimage, 19, 1194-1204. http://dx.doi.org/10.1016/S1053-8119(03)00161-7

Temple, E. (2002). Brain Mechanisms in Normal and Dyslexic readers. Current Opinion in Neurobiology, 12, 178-183. http://dx.doi.org/10.1016/S0959-4388(02)00303-3

Thomason, M., \& Thompson, P. (2011). Diffusion Imaging, White Matter and Psychopathology. Annual Review of Clinical Psychology, 7, 63-85. http://dx.doi.org/10.1146/annurev-clinpsy-032210-104507

Toplak, M. E., Jain, U., \& Tannock, R. (2005). Executive and Motivational Processes in Adolescents with Attention-DeficitHyperactivity Disorder (ADHD). Behavioral and Brain Functions, 1, 8-20. http://dx.doi.org/10.1186/1744-9081-1-8

Trauzettel-Klosinski, S., Dürrwächter, U., Klosinski, G., \& Braun, C. (2006). Cortical Activation during Word Reading and Picture Naming in Dyslexic and Non-Reading-Impaired Children. Clinical Neurophysiology, 117, 1085-1097. http://dx.doi.org/10.1016/j.clinph.2006.01.012

Trzesniewski, K. H., Moffitt, T. E., Caspi, A., Taylor, A., \& Maughan, B. (2006). Revisiting the Association between Reading Achievement and Antisocial Behavior: New Evidence of an Environmental Explanation from a Twin Study. Child Development, 77, 72-88. http://dx.doi.org/10.1111/j.1467-8624.2006.00857.x

Valdois, S., Bosse, M., \& Tainturier, M. (2004). The Cognitive Deficits Responsible for Developmental Dyslexia: Review of Evidence for a Selective Visual Attentional Disorder. Dyslexia, 10, 339-363. http://dx.doi.org/10.1002/dys.284

Vandermosten, M., Boets, B., Wouters, J., \& Ghesquiere, P. (2012). A Qualitative and Quantitative Review of Diffusion Tensor Imaging Studies in Reading and Dyslexia. Neuroscience and Biobehavioral Reviews, 36, 1532-1552. http://dx.doi.org/10.1016/j.neubiorev.2012.04.002

Vellutino, F., Fletcher, J., Snowling, M., \& Scanlon, D. (2004). Specific Reading Disability (Dyslexia): What Have We Learned in the Past Four Decades? Journal of Child Psychology and Psychiatry and Allied Disciplines, 45, 2-40. http://dx.doi.org/10.1046/j.0021-9630.2003.00305.x

von Plessen, K., Lundervold, A., Duta, N., Heiervang, E., Klauschen, F., Smievoll, A. I. et al. (2002). Less Developed Corpus Callosum in Dyslexic Subjects—A Structural MRI Study. Neuropsychologia, 40, 1035-1044.

http://dx.doi.org/10.1016/S0028-3932(01)00143-9

Wechsler, D. (1999). Wechsler Abbreviated Scale of Intelligence. San Antonio, Texas: Pearson Corporation. 
Wehner, D. T., Ahlfors, S. P., \& Mody, M. (2007). Effects of Phonological Contrast on Auditory Word Discrimination in Children with and without Reading Disability: A Magnetoencephalography (MEG) Study. Neuropsychologia, 45, 32513262. http://dx.doi.org/10.1016/j.neuropsychologia.2007.06.018

Weiss, S., \& Mueller, H. M. (2003). The Contribution of EEG Coherence to the Investigation of Language. Brain and Language, 85, 325-343. http://dx.doi.org/10.1016/S0093-934X(03)00067-1

Wilkinson, G. S., \& Robertson, G. J. (2006). Wide Range Achievement Test 4 Professional Manual. Lutz, FL: Psychological Assessment Resources.

Willcutt, E. G., Betjemann, R. S., McGrath, L. M., Chhabildas, N. A., Olson, R. K., DeFries, J. C. et al. (2010b). Etiology and Neuropsychology of Comorbidity between RD and ADHD: The Case for Multiple-Deficit Models. Cortex, 46, 13451361. http://dx.doi.org/10.1016/j.cortex.2010.06.009

Willcutt, E., Pennington, B., Duncan, L., Smith, S., Keenan, J., Wadsworth, S. et al. (2010a). Understanding the Complex Etiologies of Developmental Disorders: Behavioral and Molecular Genetic Approaches. Journal of Developmental and Behavioral Pediatrics, 31, 533-544. http://dx.doi.org/10.1097/DBP.0b013e3181ef42a1

Wolf, R., Sambataro, F., Lohr, C., Steinbrink, C., Martin, C., \& Vasic, N. (2010). Functional Brain Network Abnormalities during Verbal Working Memory Performance in Adolescents and Young Adults with Dyslexia. Neuropsychologia, 48, 309-318. http://dx.doi.org/10.1016/j.neuropsychologia.2009.09.020

Woodcock, R. W., McGrew, K. S., \& Mather, N. (2001). Examiner's Manual. Woodcock-Johnson III Tests of Achievement. Itasca, IL: Riverside Publishing.

Zeffiro, T., \& Eden, G. (2000). The Neural Basis of Developmental Dyslexia. Annals of Dyslexia, 50, 1-30. http://dx.doi.org/10.1007/s11881-000-0015-5 
Scientific Research Publishing (SCIRP) is one of the largest Open Access journal publishers. It is currently publishing more than 200 open access, online, peer-reviewed journals covering a wide range of academic disciplines. SCIRP serves the worldwide academic communities and contributes to the progress and application of science with its publication.

Other selected journals from SCIRP are listed as below. Submit your manuscript to us via either submit@scirp.org or Online Submission Portal.
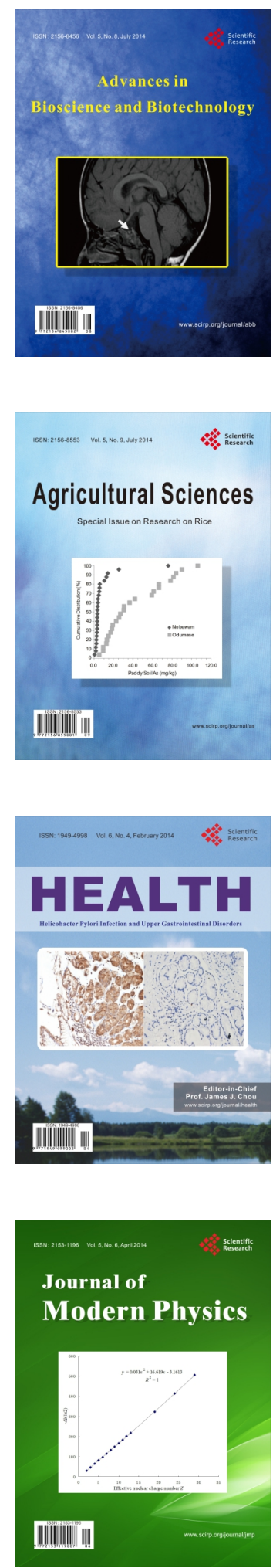
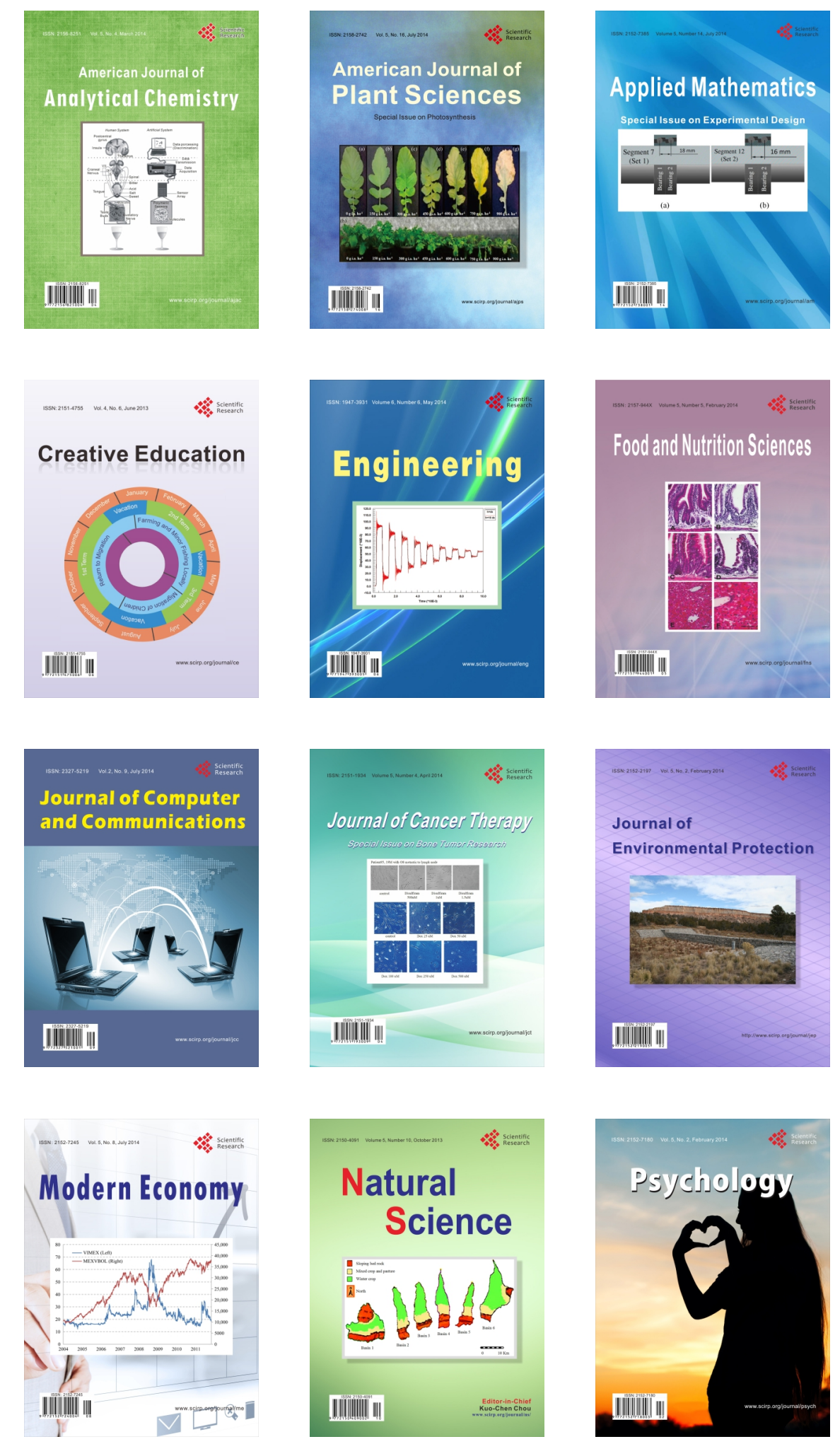